\title{
GLOBAL AND FINE APPROXIMATION OF CONVEX FUNCTIONS
}

\author{
DANIEL AZAGRA
}

Dedicated to the memory of Robb Fry

\begin{abstract}
Let $U \subseteq \mathbb{R}^{d}$ be open and convex. We prove that every (not necessarily Lipschitz or strongly) convex function $f: U \rightarrow \mathbb{R}$ can be approximated by real analytic convex functions, uniformly on all of $U$. We also show that $C^{0}$-fine approximation of convex functions by smooth (or real analytic) convex functions on $\mathbb{R}^{d}$ is possible in general if and only if $d=1$. Nevertheless, for $d \geq 2$ we give a characterization of the class of convex functions on $\mathbb{R}^{d}$ which can be approximated by real analytic (or just smoother) convex functions in the $C^{0}$-fine topology. It turns out that the possibility of performing this kind of approximation is not determined by the degree of local convexity or smoothness of the given function, but by its global geometrical behaviour. We also show that every $C^{1}$ convex and proper function on $U$ can be approximated by $C^{\infty}$ convex functions in the $C^{1}$-fine topology, and we provide some applications of these results, concerning prescription of (sub-)differential boundary data to convex real analytic functions, and smooth surgery of convex bodies.
\end{abstract}

\section{INTRODUCTION AND MAIN RESULTS}

Two important classes of functions in analysis and in geometry are those of Lipschitz functions and convex functions $f: U \subseteq \mathbb{R}^{d} \rightarrow \mathbb{R}$. Although these functions are almost everywhere differentiable (or even almost everywhere twice differentiable in the convex case), it is sometimes useful to approximate them by smooth functions which are Lipschitz or convex as well.

In the case of a Lipschitz function $f: U \subseteq \mathbb{R}^{d} \rightarrow \mathbb{R}$, this can easily be done as follows: by considering the function $x \mapsto \inf _{y \in U}\{f(y)+L|x-y|\}$ (where $L=\operatorname{Lip}(f)$, the Lipschitz constant of $f$ ), which is a Lipschitz extension of $f$ to all of $\mathbb{R}^{d}$ having the same Lipschitz constant, one can assume $U=\mathbb{R}^{d}$. Then, by setting $f_{\varepsilon}=f * H_{\varepsilon}$, where $H_{\varepsilon}(x)=\frac{1}{(4 \pi \varepsilon)^{d / 2}} \exp \left(-|x|^{2} / 4 \varepsilon\right)$ is the heat kernel, one obtains real analytic Lipschitz functions (with the same Lipschitz constants as $f$ ) which converge to $f$ uniformly on all of $\mathbb{R}^{d}$ as $\varepsilon \searrow 0$. If one replaces $H_{\varepsilon}$ with any approximate identity $\left\{\delta_{\varepsilon}\right\}_{\varepsilon>0}$ of class $C^{k}$, one obtains $C^{k}$ Lipschitz approximations. Moreover, if $\delta_{\varepsilon} \geq 0$ and $f$ is convex, then the functions $f_{\varepsilon}$ are convex as well.

Date: January 27, 2012. 
However, if $f: \mathbb{R}^{d} \rightarrow \mathbb{R}$ is convex but not globally Lipschitz, the convolutions $f * H_{\varepsilon}$ may not be well defined or, even when they are well defined, they do not converge to $f$ uniformly on $\mathbb{R}^{d}$. On the other hand, the convolutions $f * \delta_{\varepsilon}$ (where $\delta_{\varepsilon}=\varepsilon^{-d} \delta(x / \varepsilon), \delta \geq 0$ being a $C^{\infty}$ function with bounded support and $\int_{\mathbb{R}^{d}} \delta=1$ ) are always well defined, but they only provide uniform approximation of $f$ on compact sets. Now, partitions of unity cannot be used to glue these local convex approximations into a global approximation, because they do not preserve convexity. To see why this is so, let us consider the simple case of a $C^{2}$ convex function $f: \mathbb{R} \rightarrow \mathbb{R}$, to be approximated by $C^{\infty}$ convex functions. Take two bounded intervals $I_{1} \subset I_{2}$, and $C^{\infty}$ functions $\theta_{1}, \theta_{2}: \mathbb{R} \rightarrow[0,1]$ such that $\theta_{1}+\theta_{2}=1$ on $\mathbb{R}, \theta_{1}=1$ on $I_{1}$, and $\theta_{2}=1$ on $\mathbb{R} \backslash I_{2}$. Given $\varepsilon_{j}>0$ one may find $C^{\infty}$ convex functions $g_{j}$ such that $\max \left\{\left|f-g_{j}\right|,\left|f^{\prime}-g_{j}^{\prime}\right|,\left|f^{\prime \prime}-g_{j}^{\prime \prime}\right|\right\} \leq \varepsilon_{j}$ on $I_{j}$. If $g=\theta_{1} g_{1}+\theta_{2} g_{2}$ one has

$$
g^{\prime \prime}=g_{1}^{\prime \prime} \theta_{1}+g_{2}^{\prime \prime} \theta_{2}+2\left(g_{1}^{\prime}-g_{2}^{\prime}\right) \theta_{1}^{\prime}+\left(g_{1}-g_{2}\right) \theta_{1}^{\prime \prime} .
$$

If $f^{\prime \prime}>0$ on $I_{2}$ then by choosing $\varepsilon_{i}$ small enough one can control this sum and get $g^{\prime \prime} \geq 0$, but if the $g_{i}^{\prime \prime}=0$ vanish somewhere there is no way to do this (even if we managed to have $g_{2} \geq g_{1}$ and $g_{2}^{\prime} \geq g_{1}^{\prime}$, as $\theta_{1}^{\prime \prime}$ must change signs).

In [14, 15], 16] Greene and Wu studied the question of approximating a convex function defined on a (finite-dimensional) Riemannian manifold $M \mathbb{1}$, and they showed that if $f: M \rightarrow \mathbb{R}$ is strongly convex (in the sense of the following definition), then for every $\varepsilon>0$ one can find a $C^{\infty}$ strongly convex function $g$ such that $|f-g| \leq \varepsilon$ on all of $M$.

Definition 1. A $C^{2}$ function $\varphi: M \rightarrow \mathbb{R}$ is called strongly convex if its second derivative along any nonconstant geodesic is strictly positive everywhere on the geodesic. A (not necessarily smooth) function $f: M \rightarrow \mathbb{R}$ is said to be strongly convex provided that for every $p \in M$ there exists an open neighbourhood $V$ of $p$ and a strongly convex function $\varphi \in C^{2}(V)$ such that $f-\varphi$ is convex on $V 2$

This solves the problem when the given function $f$ is strongly convex. However, as Greene and $\mathrm{Wu}$ pointed out, their method cannot be used when $f$ is not strongly convex. This is inconvenient because strong convexity is a

\footnotetext{
${ }^{1}$ In Riemannian geometry convex functions have been used, for instance, in the investigation of the structure of noncompact manifolds of positive curvature by Cheeger, Greene, Gromoll, Meyer, Siohama, Wu and others, see [17, [7, 12, [13, [15, 16. The existence of global convex functions on a Riemannian manifold has strong geometrical and topological implications. For instance [12, every two-dimensional manifold which admits a global convex function that is locally nonconstant must be diffeomorphic to the plane, the cylinder, or the open Möbius strip.

${ }^{2}$ We warn the reader that, in Greene and Wu's papers, what we have just defined as strong convexity is called strict convexity. We have changed their terminology since we will be mainly concerned with the case $M=\mathbb{R}^{d}$, where one traditionally defines a strictly convex function as a function $f$ satisfying $f((1-t) x+t y)<(1-t) f(x)+t f(y)$ if $0<t<1$.
} 
very strong condition: for instance, the function $f(x)=x^{4}$ is strictly convex, but not strongly convex on any neighbourhood of 0 . However, as shown by Smith in [23, this is a necessary condition in the general Riemannian setting: for each $k=0,1, \ldots, \infty$, there exists a flat Riemannian manifold $M$ such that on $M$ there is a $C^{k}$ convex function which cannot be globally approximated by a $C^{k+1}$ convex function (here $C^{\infty+1}$ means real analytic). There are no results characterizing the manifolds on which global approximation of convex functions by smooth convex functions is possible. Even in the most basic case $M=\mathbb{R}^{d}$, we have been unable to find any reference dealing with the problem of finding smooth global approximations of (not necessarily Lipschitz or strongly) convex functions.

One of the main purposes of this paper is proving the following.

Theorem 1. Let $U \subseteq \mathbb{R}^{d}$ be open and convex. For every convex function $f: U \rightarrow \mathbb{R}$ and every $\varepsilon>0$ there exists a real-analytic convex function $g: U \rightarrow \mathbb{R}$ such that $f-\varepsilon \leq g \leq f$.

This result is optimal in several ways: as we will see, it is not possible to obtain $C^{0}$-fine approximation of convex functions by $C^{1}$ convex functions on $\mathbb{R}^{d}$ when $d \geq 2$ (and even in the case $d=1$ this kind of approximation is not possible from below).

In showing this theorem we will develop a gluing technique for convex functions which will prove to be useful also in the setting of Riemannian manifolds or Banach spaces.

Definition 2. Let $X$ be $\mathbb{R}^{d}$, or a complete Riemannian manifold (not necessarily finite-dimensional), or a Banach space, and let $U \subseteq X$ be open and convex. We will say that a continuous convex function $f: U \rightarrow \mathbb{R}$ can be approximated from below by $C^{k}$ convex functions, uniformly on bounded subsets of $U$, provided that for every bounded set $B$ with $\bar{B} \subset U$ and $\operatorname{dist}(B, \partial U)>0$, and for every $\varepsilon>0$ there exists a $C^{k}$ convex function $g: U \rightarrow \mathbb{R}$ such that

(1) $g \leq f$ on $U$, and

(2) $f-\varepsilon \leq g$ on $B$.

(In the case when $U=X$ is unbounded we will use the convention that $\operatorname{dist}(B, \partial U)=\infty$ for every bounded set $B \subset X$.)

Theorem 2 (Gluing convex approximations). Let $X$ be $\mathbb{R}^{d}$, or a complete Riemannian manifold (not necessarily finite-dimensional), or a $B a$ nach space, and let $U \subseteq X$ be open and convex. Assume that $U=\bigcup_{n=1}^{\infty} B_{n}$, where the $B_{n}$ are open bounded convex sets such that $\operatorname{dist}\left(B_{n}, \partial U\right)>0$ and $\overline{B_{n}} \subset B_{n+1}$ for each $n$. Assume also that $U$ has the property that every continuous, convex function $f: U \rightarrow \mathbb{R}$ can be approximated from below by $C^{k}$ convex (resp. strongly convex) functions $(k \in \mathbb{N} \cup\{\infty\}$ ), uniformly on bounded subsets of $U$.

Then every continuous convex function $f: U \rightarrow \mathbb{R}$ can be approximated from below by $C^{k}$ convex (resp. strongly convex) functions, uniformly on $U$. 
From this result (and from its proof and the known results on approximation on bounded sets) we will easily deduce the following corollaries.

Corollary 1. Let $U \subseteq \mathbb{R}^{d}$ be open and convex. For every convex function $f: U \rightarrow \mathbb{R}$ and every $\varepsilon>0$ there exists a $C^{\infty}$ convex function $g: U \rightarrow \mathbb{R}$ such that $f-\varepsilon \leq g \leq f$. Moreover $g$ can be taken so as to preserve local Lipschitz constants of $f$ (meaning $\operatorname{Lip}\left(g_{\left.\right|_{B}}\right) \leq \operatorname{Lip}\left(f_{\left.\right|_{(1+\varepsilon) B}}\right)$ for every ball $B \subset U$ ). And if $f$ is strictly (or strongly) convex, so can $g$ be chosen.

Corollary 2. Let $M$ be a Cartan-Hadamard Riemannian manifold (not necessarily finite dimensional), and $U \subseteq M$ be open and convex. For every convex function $f: U \rightarrow \mathbb{R}$ which is bounded on bounded subsets $B$ of $U$ with $\operatorname{dist}(B, \partial U)>0$, and for every $\varepsilon>0$ there exists a $C^{1}$ convex function $g: U \rightarrow \mathbb{R}$ such that $f-\varepsilon \leq g \leq f$. Moreover $g$ can be chosen so as to preserve the set of minimizers and the local Lipschitz constants of $f$. And, if $f$ is strictly convex, so can $g$ be taken.

One should expect that the above corollary is not optimal (in that approximation by $C^{\infty}$ convex functions should be possible).

Corollary 3. Let $X$ be a Banach space whose dual is locally uniformly convex, and $U \subseteq X$ be open and convex. For every convex function $f$ : $U \rightarrow \mathbb{R}$ which is bounded on bounded subsets $B$ of $U$ with $\operatorname{dist}(B, \partial U)>0$, and for every $\varepsilon>0$ there exists a $C^{1}$ convex function $g: U \rightarrow \mathbb{R}$ such that $f-\varepsilon \leq g \leq f$. Moreover $g$ can be taken so as to preserve the set of minimizers and the local Lipschitz constants of $f$. And if $f$ is strictly convex, so can $g$ be taken.

A question remains open whether every convex function $f$ defined on a separable infinite-dimensional Hilbert space $X$ which is bounded on bounded sets can be globally approximated by $C^{2}$ convex functions (notice that Theorem 2 cannot be combined with the results of [8], [9] on smooth and real analytic approximation of bounded convex bodies in Banach spaces in order to give a solution to this problem. Although one can use these results, together with the implicit function theorem, to find smooth convex approximations of $f$ on a bounded set, the approximating functions obtained by this process are not defined on all of $X$ and are not strongly convex, hence it is not clear how to extend them to a smooth convex function below $f$ on $X$, or even if this should be possible at all).

As a byproduct of the proof of Theorem 11 we will also obtain the following characterization of the class of convex functions that can be globally approximated by strongly convex functions on $\mathbb{R}^{d}$.

Proposition 1. Let $f: \mathbb{R}^{d} \rightarrow \mathbb{R}$ be a convex function. The following conditions are equivalent:

(1) $f$ cannot be uniformly approximated by strictly convex functions.

(2) $f$ cannot be uniformly approximated by strongly convex functions. 
(3) There exist $k<d$, a linear projection $P: \mathbb{R}^{d} \rightarrow \mathbb{R}^{k}$, a convex function $c: \mathbb{R}^{k} \rightarrow \mathbb{R}$ and a linear function $\ell: \mathbb{R}^{d} \rightarrow \mathbb{R}$ such that $f=c \circ P+\ell$.

We will also consider fine approximation of convex functions on subsets of $\mathbb{R}^{d}$. In this direction, the only known results concerning $C^{0}$-fine approximation of convex functions by smooth convex functions are also due to Greene and $\mathrm{Wu}$ [16], who showed that every strongly convex function $f$ defined on a (finite-dimensional) Riemannian manifold $M$ can be approximated by $C^{\infty}$ strongly convex functions in the $C^{0}$-fine topology.

We say that a convex function $f \in C^{k}(M)$ can be approximated by $C^{\infty}$ convex functions in the $C^{k}$-fine topology provided that for every continuous function $\varepsilon: M \rightarrow(0, \infty)$ there exists a convex function $g \in C^{\infty}(M)$ such that $|f-g| \leq \varepsilon$ and $\left\|D^{j} f-D^{j} g\right\| \leq \varepsilon$ on $M$ for $j \leq k$ when $k \geq 1$.

For $d=1$ we have the following.

Theorem 3. Let $U \subseteq \mathbb{R}$ be an open interval. Every convex function $f$ : $U \rightarrow \mathbb{R}$ can be approximated by real analytic convex functions in the $C^{0}$-fine topology.

For $d \geq 2$, we will provide a characterization of the class of convex functions on $\mathbb{R}^{d}$ which can be approximated in the $C^{0}$-fine topology by smoother (or real analytic) convex functions. Interestingly, the possibility of performing this kind of approximation has very little to do with the degree of local convexity or smoothness of the given function. It is the global geometrical behaviour of the function that determines whether or not it can be approximated by more regular convex functions in this topology.

Definition 3. Let $U \subseteq \mathbb{R}^{d}$ be open and convex. We will say that a function $f: U \rightarrow \mathbb{R}$ is properly convex provided that $f=\ell+c$, where $\ell$ is linear, $c: U \mapsto[a, b),-\infty<a<b \leq \infty$, and $c$ is convex and proper (meaning that $c^{-1}[a, \beta]$ is compact for every $\left.\beta \in[a, b)\right)$.

It is obvious that proper convexity is not a restrictive property from a local point of view, but it has global geometrical implications.

Theorem 4. Let $f: \mathbb{R}^{d} \rightarrow \mathbb{R}$ be a $C^{p}$ convex function which is not of class $C^{p+1}, p \in \mathbb{N} \cup\{\infty\}, d \geq 2$. The following statements are equivalent:

(1) $f$ is properly convex.

(2) $f$ can be written in the form $f=\ell+c$, where $\ell$ is linear and $\lim _{|x| \rightarrow \infty} c(x)=\infty$.

(3) $f$ cannot be written in the form $f=c \circ P+\ell$, where $P: \mathbb{R}^{d} \rightarrow \mathbb{R}^{k}$ is a linear projection, $k<d, c: \mathbb{R}^{k} \rightarrow \mathbb{R}$ is convex, and $\ell: \mathbb{R}^{d} \rightarrow \mathbb{R}$ is linear.

(4) $f$ can be approximated by strongly convex real analytic functions in the $C^{0}$-fine topology.

(5) $f$ can be approximated by $C^{p+1}$ convex functions in the $C^{0}$-fine topology. 
In the case when $U$ is a proper open convex subset of $\mathbb{R}^{d}, d \geq 2$, it would be harder to establish a full characterization (in the spirit of the preceding theorem) of the class of convex functions $f: U \rightarrow \mathbb{R}$ which can be approximated by smoother convex functions. We do not embark on such a program, but we do prove that every properly convex function on $U$ can be approximated by convex real analytic functions in the $C^{0}$-fine topology.

Theorem 5. Let $U \subseteq \mathbb{R}^{d}$ be open and convex, and $f: U \rightarrow \mathbb{R}$ be properly convex. Then $f$ can be approximated by strongly convex real analytic functions in the $C^{0}$-fine topology.

When $f \in C^{1}$, we will show a slightly weaker result (but still powerful enough to imply quite interesting geometrical corollaries): we are able to approximate any $C^{1}$ properly convex function by $C^{\infty}$ convex functions in the $C^{1}$-fine topology.

Theorem 6. Let $U \subseteq \mathbb{R}^{d}$ be open and convex, and $f: U \rightarrow \mathbb{R}$ be properly convex and $C^{1}$. Then $f$ can be approximated by $C^{\infty}$ convex functions in the $C^{1}$-fine topology.

We will also show (see Example 3 below) that on $(-1,1) \times(-1,1) \subset \mathbb{R}^{2}$ there exists a $C^{p}$, but not $C^{p+1}$, convex function $f$ which is affine exactly on a very thin neighbourhood of a line, which is strongly convex outside a very small neighbourhood of this line, and which cannot be approximated by $C^{p+1}$ convex functions in the $C^{0}$-fine topology. Hence, even in the case $U \neq \mathbb{R}^{d}$, proper convexity is a very reasonable condition to require of a nonsmooth convex function, if one wants to approximate it by smooth convex functions.

As a first geometrical application of Theorem 5, we will show that one can sometimes prescribe subdifferential data to real analytic convex functions at the boundary of a compact convex body.

Corollary 4. Let $U \subseteq \mathbb{R}^{d}$ be open and convex, $f: U \rightarrow \mathbb{R}$ be a convex function of the form $f=\ell+c$, where $\ell$ is linear and $c$ is proper, $K a$ compact convex body of the form $K=c^{-1}(-\infty, b]$, and $\varepsilon: \operatorname{int}(\mathrm{K}) \rightarrow(0, \infty)$ a continuous function. Then there exists a convex function $F: U \rightarrow \mathbb{R}$ such that

(1) $F=f$ on $U \backslash \operatorname{int}(K)$

(2) $|F-f| \leq \varepsilon$ on $\operatorname{int}(K)$

(3) $F$ is strongly convex and real analytic on int $(K)$

(4) $\partial F(x)=\partial f(x)$ for each $x \in \partial K$.

Moreover, if $f \in C^{1}(U \backslash$ int $(K))$ then $F \in C^{1}(U)$.

As is usual, we denote $\partial F(x)=\left\{\zeta: \mathbb{R}^{n} \rightarrow \mathbb{R} \mid \zeta\right.$ is linear , $F(y)-F(x) \geq$ $\zeta(x-y)$ for all $y \in U\}$, the subdifferential of $F$.

In the case when the given function $f$ is already $C^{2}$ outside $\operatorname{int}(K)$, we will also show the following.

Corollary 5. Let $U \subseteq \mathbb{R}^{d}$ be open and convex, $f: U \rightarrow \mathbb{R}$ be a convex function of the form $f=\ell+c$, where $\ell$ is linear and $c$ is proper, $K a$ 
compact convex body of the form $K=c^{-1}(-\infty, b]$, and $\varepsilon: \operatorname{int}(\mathrm{K}) \rightarrow(0, \infty)$ a continuous function. Assume that $f$ is $C^{2}$ on $U \backslash$ int $(K)$. Then there exists a $C^{2}$ convex function $F: U \rightarrow \mathbb{R}$ such that

(1) $F=f$ on $U \backslash \operatorname{int}(K)$

(2) $|F-f| \leq \varepsilon$ on $\operatorname{int}(K)$

(3) $F$ is $C^{\infty}$ on $\operatorname{int}(K)$.

These corollaries are in the spirit of Ghomi's work on optimal smoothing of convex functions [10], but note that here we do not require strong convexity of $f$ on any neighbourhood of $\partial K$.

The above corollaries are also useful in smooth surgery of convex bodies, e.g. as in the following situation: one has a convex body with a relatively small part that one does not like (for instance because it is not sufficiently smooth or convex). Assuming that the part is a intersection of the given body with a half-space, then one can replace that part with another piece which approximates the given part, and which has a smooth boundary, with no loss of first or second order differential information at the seam.

Corollary 6. Let $C$ be a compact convex body in $\mathbb{R}^{d}$, and let $K$ be a convex body of the form $K=\ell^{-1}(-\infty, b] \cap C$, where $\ell$ is a linear function on $\mathbb{R}^{d}$. Let $P$ be the orthogonal projection of $\mathbb{R}^{d}$ onto the subspace Kerl, and assume that $P(K)$ is contained in the interior of $P(C)$, and that $\partial C \backslash \operatorname{int}(K)$ is contained in a $C^{p}$ convex hypersurface, $p=1,2$. Then, for every number $\varepsilon>0$ there exists a compact convex body $D$ such that:

(1) $\partial D$ is a compact $C^{p}$ convex hypersurface;

(2) $C \backslash K=D \backslash K$;

(3) $\partial D \cap \ell^{-1}(-\infty, b)$ is a $C^{\infty}$ convex hypersurface (or even real analytic and strongly convex in the case $p=1)$;

(4) $\operatorname{dist}\left(\partial \mathrm{C} \cap \ell^{-1}(-\infty, \mathrm{b}], \partial \mathrm{D} \cap \ell^{-1}(-\infty, \mathrm{b}]\right) \leq \varepsilon$.

One might like to compare the above corollary with the main result of [11, which provides a procedure for smoothing the edges and vertices of a convex polytope.

\section{A general GLuing technique}

In order to prove Theorem 2 we will use the following.

Lemma 1 (Smooth maxima). For every $\varepsilon>0$ there exists a $C^{\infty}$ function $M_{\varepsilon}: \mathbb{R}^{2} \rightarrow \mathbb{R}$ with the following properties:

(1) $M_{\varepsilon}$ is convex;

(2) $\max \{x, y\} \leq M_{\varepsilon}(x, y) \leq \max \{x, y\}+\frac{\varepsilon}{2}$ for all $(x, y) \in \mathbb{R}^{2}$.

(3) $M_{\varepsilon}(x, y)=\max \{x, y\}$ whenever $|x-y| \geq \varepsilon$.

(4) $M_{\varepsilon}(x, y)=M_{\varepsilon}(y, x)$.

(5) $\operatorname{Lip}\left(M_{\varepsilon}\right)=1$ with respect to the norm $\|\cdot\|_{\infty}$ in $\mathbb{R}^{2}$.

(6) $y-\varepsilon \leq x<x^{\prime} \Longrightarrow M_{\varepsilon}(x, y)<M_{\varepsilon}\left(x^{\prime}, y\right)$.

(7) $x-\varepsilon \leq y<y^{\prime} \Longrightarrow M_{\varepsilon}(x, y)<M_{\varepsilon}\left(x, y^{\prime}\right)$. 
(8) $x \leq x^{\prime}, y \leq y^{\prime} \Longrightarrow M_{\varepsilon}(x, y) \leq M_{\varepsilon}\left(x^{\prime}, y^{\prime}\right)$, with a strict inequality in the case when both $x<x^{\prime}$ and $y<y^{\prime}$.

We will call $M_{\varepsilon}$ a smooth maximum.

Proof. It is easy to construct a $C^{\infty}$ function $\theta: \mathbb{R} \rightarrow(0, \infty)$ such that:

(1) $\theta(t)=|t|$ if and only if $|t| \geq \varepsilon$;

(2) $\theta$ is convex and symmetric;

(3) $\operatorname{Lip}(\theta)=1$.

Then it is also easy to check that the function $M_{\varepsilon}$ defined by

$$
M_{\varepsilon}(x, y)=\frac{x+y+\theta(x-y)}{2}
$$

satisfies the required properties. For instance, let us check properties (v), (vi), (vii) and (viii), which are perhaps less obvious than the others. Since $\theta$ is 1-Lipschitz we have

$$
\begin{aligned}
& M_{\varepsilon}(x, y)-M_{\varepsilon}\left(x^{\prime}, y^{\prime}\right)=\frac{x-x^{\prime}+y-y^{\prime}+\theta(x-y)-\theta\left(x^{\prime}-y^{\prime}\right)}{2} \leq \\
& \frac{(x-x)^{\prime}+\left(y-y^{\prime}\right)+\left|x-x^{\prime}-y+y^{\prime}\right|}{2}= \\
& \max \left\{x-x^{\prime}, y-y^{\prime}\right\} \leq \max \left\{\left|x-x^{\prime}\right|,\left|y-y^{\prime}\right|\right\},
\end{aligned}
$$

which establishes (v). To verify (vi) and (vii), note that our function $\theta$ must satisfy $\left|\theta^{\prime}(t)\right|<1 \Longleftrightarrow|t|<\varepsilon$. Then we have

$\frac{\partial M_{\varepsilon}}{\partial x}(x, y)=\frac{1}{2}\left(1+\theta^{\prime}(x-y)\right) \geq \frac{1}{2}\left(1-\left|\theta^{\prime}(x-y)\right|\right)>0$ whenever $|x-y|<\varepsilon$, while

$$
\frac{\partial M_{\varepsilon}}{\partial x}(x, y)=\frac{1}{2}\left(1+\theta^{\prime}(x-y)\right)= \begin{cases}1, & \text { if } x \geq y+\varepsilon \\ 0, & \text { if } y \geq x+\varepsilon\end{cases}
$$

This implies (vi) and, together with (iv), also (vii) and the first part of (viii). Finally, if for instance we have $x^{\prime}>x=\max \{x, y\}$ then $M_{\varepsilon}(x, y)<$ $M_{\varepsilon}\left(x^{\prime}, y\right)$ by (vi), and if in addition $y^{\prime}>y$ then $M_{\varepsilon}\left(x^{\prime}, y\right) \leq M_{\varepsilon}\left(x^{\prime}, y^{\prime}\right)$ by the first part of (viii), hence $M_{\varepsilon}(x, y)<M_{\varepsilon}\left(x^{\prime}, y^{\prime}\right)$. This shows the second part of (viii).

The smooth maxima $M_{\varepsilon}$ are useful to approximate the maximum of two functions without losing convexity or other key properties of the functions, as we next see.

Proposition 2. Let $U \subseteq X$ be as in the statement of Theorem $\mathbb{Q}, M_{\varepsilon}$ as in the preceding Lemma, and let $f, g: U \rightarrow \mathbb{R}$ be convex functions. For every $\varepsilon>0$, the function $M_{\varepsilon}(f, g): U \rightarrow \mathbb{R}$ has the following properties:

(1) $M_{\varepsilon}(f, g)$ is convex.

(2) If $f$ is $C^{k}$ on $\{x: f(x) \geq g(x)-\varepsilon\}$ and $g$ is $C^{k}$ on $\{x: g(x) \geq$ $f(x)-\varepsilon\}$ then $M_{\varepsilon}(f, g)$ is $C^{k}$ on $U$. In particular, if $f, g$ are $C^{k}$, then so is $M_{\varepsilon}(f, g)$.

(3) $M_{\varepsilon}(f, g)=f$ if $f \geq g+\varepsilon$. 
(4) $M_{\varepsilon}(f, g)=g$ if $g \geq f+\varepsilon$.

(5) $\max \{f, g\} \leq M_{\varepsilon}(f, g) \leq \max \{f, g\}+\varepsilon / 2$.

(6) $M_{\varepsilon}(f, g)=M_{\varepsilon}(g, f)$.

(7) $\operatorname{Lip}\left(M_{\varepsilon}(f, g)_{\left.\right|_{B}}\right) \leq \max \left\{\operatorname{Lip}\left(f_{\left.\right|_{B}}\right), \operatorname{Lip}\left(g_{\left.\right|_{B}}\right)\right\}$ for every ball $B \subset U$ (in particular $M_{\varepsilon}(f, g)$ preserves common local Lipschitz constants of $f$ and $g$ ).

(8) If $f, g$ are strictly convex on a set $B \subseteq U$, then so is $M_{\varepsilon}(f, g)$.

(9) If $f, g \in C^{2}(X)$ are strongly convex on a set $B \subseteq U$, then so is $M_{\varepsilon}(f, g)$.

(10) If $f_{1} \leq f_{2}$ and $g_{1} \leq g_{2}$ then $M_{\varepsilon}\left(f_{1}, g_{1}\right) \leq M_{\varepsilon}\left(f_{2}, g_{2}\right)$.

Proof. Properties (ii), (iii), (iv), (v), (vi), (vii) and (x) are obvious from the preceding lemma. To check (i) and (viii), we simply use (x) and convexity of $f, g$ and $M_{\varepsilon}$ to see that, for $x, y \in U, t \in[0,1]$,

$$
\begin{aligned}
& M_{\varepsilon}(f(t x+(1-t) y), g(t x+(1-t) y)) \leq \\
& M_{\varepsilon}(t f(x)+(1-t) f(y), t g(x)+(1-t) g(y))= \\
& M_{\varepsilon}(t(f(x), g(x))+(1-t)(f(y), g(y))) \leq \\
& t M_{\varepsilon}(f(x), g(x))+(1-t) M_{\varepsilon}(f(y), g(y)),
\end{aligned}
$$

and, according to (viii) in the preceding lemma, the first inequality is strict whenever $f, g$ are strictly convex and $0<t<1$. To check (ix), it is sufficient to see that the function $t \mapsto M_{\varepsilon}(f, g)(\gamma(t))$ has a strictly positive second derivative at each $t$, where $\gamma(t)=x+t v$ with $v \neq 0$ (or, in the Riemannian case, $\gamma$ is a nonconstant geodesic). So, by replacing $f, g$ with $f(\gamma(t))$ and $g(\gamma(t))$ we can assume that $f$ and $g$ are defined on an interval $I \subseteq \mathbb{R}$ on which we have $f^{\prime \prime}(t)>0, g^{\prime \prime}(t)>0$. But in this case we easily compute

$$
\begin{aligned}
& \frac{d^{2}}{d t^{2}} M_{\varepsilon}(f(t), g(t))= \\
& \frac{\left(1+\theta^{\prime}(f(t)-g(t))\right) f^{\prime \prime}(t)+\left(1-\theta^{\prime}(f(t)-g(t))\right) g^{\prime \prime}(t)}{2}+\frac{\theta^{\prime \prime}(f(t)-g(t))(f(t)-g(t))^{2}}{2} \geq \\
& \geq \frac{1}{2} \min \left\{f^{\prime \prime}(t), g^{\prime \prime}(t)\right\}>0,
\end{aligned}
$$

because $\left|\theta^{\prime}\right| \leq 1$ and $\theta^{\prime \prime} \geq 0$.

\section{Proof of Theorem 2}

Given a continuous convex function $f: U \rightarrow \mathbb{R}$ and $\varepsilon>0$, we start defining $f_{1}=f$ and use the assumption that $f_{1}-\varepsilon / 2$ can be approximated from below by $C^{k}$ convex functions, to find a $C^{k}$ convex function $h_{1}: U \rightarrow \mathbb{R}$ such that

$$
f_{1}-\varepsilon \leq h_{1} \text { on } B_{1} \text {, and } h_{1} \leq f_{1}-\frac{\varepsilon}{2} \text { on } U \text {. }
$$


We put $g_{1}=h_{1}$. Now define $f_{2}=f_{1}-\varepsilon$ and find a convex function $h_{2} \in$ $C^{k}(U)$ such that

$$
f_{2}-\frac{\varepsilon}{2} \leq h_{2} \text { on } B_{2}, \text { and } h_{2} \leq f_{2}-\frac{\varepsilon}{4} \text { on } U .
$$

Set

$$
g_{2}=M_{\frac{\varepsilon}{10^{2}}}\left(g_{1}, h_{2}\right) .
$$

By the preceding proposition we know that $g_{2}$ is a convex $C^{k}$ function satisfying

and

$$
\max \left\{g_{1}, h_{2}\right\} \leq g_{2} \leq \max \left\{g_{1}, h_{2}\right\}+\frac{\varepsilon}{10^{2}} \text { on } U,
$$

$$
g_{2}(x)=\max \left\{g_{1}(x), h_{2}(x)\right\} \text { whenever }\left|h_{1}(x)-h_{2}(x)\right| \geq \frac{\varepsilon}{10^{2}} .
$$

Claim 1. We have

$$
g_{2}=g_{1} \text { on } B_{1}, \quad \text { and } \quad f-\varepsilon-\frac{\varepsilon}{2} \leq g_{2} \leq f-\frac{\varepsilon}{2}+\frac{\varepsilon}{10^{2}} \text { on } B_{2} .
$$

Indeed, if $x \in B_{1}$,

$$
g_{1}(x) \geq f_{1}(x)-\varepsilon=f_{2}(x)-\frac{\varepsilon}{4}+\frac{\varepsilon}{4} \geq h_{2}(x)+\frac{\varepsilon}{4} \geq h_{2}(x)+\frac{\varepsilon}{10^{2}},
$$

hence $g_{2}(x)=g_{1}(x)$, and in particular $f(x)-\frac{\varepsilon}{2} \geq g_{2}(x) \geq f(x)-\varepsilon$. While, if $x \in B_{2} \backslash B_{1}$ then

$$
\begin{aligned}
& f(x)-\varepsilon-\frac{\varepsilon}{2} \leq \max \left\{g_{1}(x), h_{2}(x)\right\} \leq g_{2}(x) \leq \max \left\{g_{1}(x), h_{2}(x)\right\}+\frac{\varepsilon}{10^{2}} \leq \\
& \max \left\{f(x)-\frac{\varepsilon}{2}, f(x)-\varepsilon-\frac{\varepsilon}{4}\right\}+\frac{\varepsilon}{10^{2}}=f(x)-\frac{\varepsilon}{2}+\frac{\varepsilon}{10^{2}} .
\end{aligned}
$$

This proves the claim.

Next, define $f_{3}=f_{2}-\varepsilon / 2=f-\varepsilon-\varepsilon / 2$, find a convex $C^{k}$ function $h_{3}$ on $U$ so that

$$
f_{3}-\frac{\varepsilon}{2^{2}} \leq h_{3} \text { on } B_{3}, \text { and } h_{3} \leq f_{3}-\frac{\varepsilon}{2^{3}} \text { on } U
$$

and set

$$
g_{3}=M_{\frac{\varepsilon}{10^{3}}}\left(g_{2}, h_{3}\right)
$$

Claim 2. We have

$g_{3}=g_{2}$ on $B_{2}, \quad$ and $f-\varepsilon-\frac{\varepsilon}{2}-\frac{\varepsilon}{2^{2}} \leq g_{3} \leq f-\frac{\varepsilon}{2}+\frac{\varepsilon}{10^{2}}+\frac{\varepsilon}{10^{3}}$ on $B_{3}$.

This is easily checked as before.

In this fashion we can inductively define a sequence of $C^{k}$ convex functions $g_{n}$ on $U$ such that

$$
\begin{gathered}
g_{n}=g_{n-1} \text { on } B_{n-1}, \quad \text { and } \\
f-\varepsilon-\frac{\varepsilon}{2}-\frac{\varepsilon}{2^{2}}-\ldots-\frac{\varepsilon}{2^{n-1}} \leq g_{n} \leq f-\frac{\varepsilon}{2}+\frac{\varepsilon}{10^{2}}+\frac{\varepsilon}{10^{3}}+\ldots+\frac{\varepsilon}{10^{n}} \text { on } B_{n}
\end{gathered}
$$

(at each step of the inductive process we define $f_{n}=f_{n-1}-\varepsilon / 2^{n-2}=$ $f-\varepsilon-\ldots-\varepsilon / 2^{n-2}$, we find $h_{n}$ convex and $C^{k}$ such that $f_{n}-\varepsilon / 2^{n-1} \leq h_{n}$ on $B_{n}$ and $h_{n} \leq f_{n}-\varepsilon / 2^{n}$ on $U$, and we put $\left.g_{n}=M_{\varepsilon / 10^{n}}\left(g_{n-1}, h_{n}\right)\right)$. 
Having constructed a sequence $g_{n}$ with such properties, we finally define

$$
g(x)=\lim _{n \rightarrow \infty} g_{n}(x) .
$$

Since we have $g_{n+k}=g_{n}$ on $B_{n}$ for all $k \geq 1$, it is clear that $g=g_{n}$ on each $B_{n}$, which implies that $g$ is $C^{k}$ and convex on $U$ (or even strongly convex when the $g_{n}$ are strongly convex). Besides, for every $x \in U=\bigcup_{n=1}^{\infty} B_{n}$ we have

$$
f(x)-2 \varepsilon=f(x)-\sum_{n=1}^{\infty} \frac{\varepsilon}{2^{n-1}} \leq g(x) \leq f(x)-\frac{\varepsilon}{2}+\sum_{n=2}^{\infty} \frac{\varepsilon}{10^{n}},
$$

hence $f-2 \varepsilon \leq g \leq f$.

Remark 1. From the above proof and from Proposition 2 it is clear that this method of transferring convex approximations on bounded sets to global convex approximations preserves strict and strong convexity, local Lipschitzness, minimizers and order, whenever the given approximations on bounded sets have these properties.

\section{Proofs of Corollaries 1, 2] and 3}

We will deduce our corollaries by combining Theorem 2 with the known results on approximation of convex functions on bounded sets mentioned in the introduction, and with the following.

Proposition 3. Let $X$ be $\mathbb{R}^{d}$, or a Cartan-Hadamard manifold (not necessarily finite-dimensional), or a Banach space, and let $U \subseteq X$ be open and convex. Assume that $U$ has the property that every Lipschitz convex function on $U$ can be approximated by $C^{k}$ convex (resp. strongly convex) functions, uniformly on $U$.

Then every convex function $f: U \rightarrow \mathbb{R}$ which is bounded on bounded subsets $B$ of $U$ with $\operatorname{dist}(B, \partial U)>0$ can be approximated from below by $C^{k}$ convex (resp. strongly convex) functions, uniformly on bounded subsets of $U$.

Proof. It is well known that a convex function $f: U \rightarrow \mathbb{R}$ which is bounded on bounded subsets $B$ of $U$ with $\operatorname{dist}(B, \partial U)>0$ is also Lipschitz on each such subset $B$ of $X$. So let $B \subset U$ be bounded, open and convex with $\operatorname{dist}(B, \partial U)>0$, put $L=\operatorname{Lip}\left(f_{\left.\right|_{B}}\right)$, and define

$$
g(x)=\inf \{f(y)+L d(x, y): y \in U\},
$$

where $d(x, y)=\|x-y\|$ in the case when $X$ is $\mathbb{R}^{d}$ or a Banach space, and $d$ is the Riemannian distance in $X$ when $X$ is a Cartan-Hadamard manifold.

Claim 3. The function $g$ has the following properties:

(1) $g$ is convex on $X$.

(2) $g$ is L-Lipschitz on $X$.

(3) $g=f$ on $B$.

(4) $g \leq f$ on $U$ 
These are well known facts in the vector space case, but perhaps not so in the Riemannian setting, so let us say a few words about the proof. Property (iv) is obvious. To see that the reverse inequality holds on $B$, take $x \in B$ and a subdifferential $\zeta \in D^{-} f(x)$ (we refer to [3], 2] for the definitions and some properties of the Fréchet subdifferential and inf convolution on Riemannian manifolds). We have $\|\zeta\|_{x} \leq L$ because $f$ is $L$-Lipschitz on $B$. Since $\exp _{x}: T X_{x} \rightarrow X$ is a diffeomorphism, for every $y \in X$ there exists $v_{y} \in T X_{x}$ such that $\exp _{x}\left(v_{y}\right)=y$. And, because $t \mapsto f\left(\exp _{x}\left(t v_{y}\right)\right)$ is convex, we have $f\left(\exp _{x}\left(t v_{y}\right)\right)-f(x) \geq\left\langle\zeta, t v_{y}\right\rangle_{x}$ for every $t$, and in particular, taking $t=1$, we get $f(y)-f(x) \geq\left\langle\zeta, t v_{y}\right\rangle_{x} \geq-\|\zeta\|_{x}\left\|v_{y}\right\|_{x} \geq-L d(x, y)$. Hence $f(y)+L d(x, y) \geq f(x)$ for all $y \in X$, and taking the inf we get $g(x) \geq f(x)$. Therefore $g=f$ on $B$. Showing (ii) is easy (as a matter of fact this is true in every metric space). Finally, to see that $g$ is convex on $X$, one does have to use that $X$ is a Cartan-Hadamard manifold. We note that in a Cartan-Hadamard manifold $X$ the distance function $d: X \times X \rightarrow[0, \infty)$ is globally convex (see for instance [21, V.4.3] and [2, Corollary 4.2]), and that if $X \times U \ni(x, y) \mapsto F(x, y)$ is convex then $x \mapsto \inf _{y \in U} F(x, y)$ is also convex on $X$ (see [2, Lemma 3.1]). Since $(x, y) \mapsto f(y)+L d(x, y)$ is convex on $X \times U$, this shows (i).

Now, for a given $\varepsilon>0$, by assumption there exists a $C^{k}$ convex (resp. strongly convex) function $\varphi: U \rightarrow \mathbb{R}$ so that $g-\varepsilon \leq \varphi \leq g$ on $U$. Since $g \leq f$ on $U$, and $g=f$ on $B$, this implies that $\varphi \leq f$ on $U$, and $f-\varepsilon \leq \varphi$ on $B$.

Let $f: \mathbb{R}^{d} \rightarrow \mathbb{R}$ be continuous. As we recalled in the introduction, if $\delta: \mathbb{R}^{d} \rightarrow[0, \infty)$ is a $C^{\infty}$ function such that $\delta(x)=0$ whenever $\|x\| \geq 1$, and $\int_{\mathbb{R}^{d}} \delta=1$, then the functions $f_{\varepsilon}(x)=\int_{\mathbb{R}^{d}} f(x-y) \delta_{\varepsilon}(y) d y$ (where $\delta_{\varepsilon}(x)=$ $\left.\varepsilon^{-d} \delta(x / \varepsilon)\right)$ are $C^{\infty}$ and converge to $f(x)$ uniformly on every compact set, as $\varepsilon \searrow 0$. Moreover, as is well known and easily checked:

(1) If $f$ is uniformly continuous then $f_{\varepsilon}$ converges to $f$ uniformly on $\mathbb{R}^{d}$.

(2) If $f$ is convex (resp. strictly, or strongly convex), so is $f_{\varepsilon}$.

(3) If $f$ is Lipschitz, so is $f_{\varepsilon}$, and $\operatorname{Lip}\left(f_{\varepsilon}\right)=\operatorname{Lip}(f)$.

(4) If $f$ is locally $\operatorname{Lipschitz,} \operatorname{Lip}\left(f_{\varepsilon_{\left.\right|_{B}}}\right)=\operatorname{Lip}\left(f_{\left.\right|_{(1+\varepsilon) B}}\right)$ for every ball $B$.

(5) If $f \leq g$ then $f_{\varepsilon} \leq g_{\varepsilon}$.

(6) If $f$ is $C^{1}$ then $D f_{\varepsilon}$ converges to $D f$ uniformly on compact subsets of $\mathbb{R}^{d}$

Therefore this method provides uniform approximation of Lipschitz convex functions by $C^{\infty}$ convex functions, uniformly on $\mathbb{R}^{d}$. By Proposition 3 we then have that every (not necessarily Lipschitz) convex function $f: \mathbb{R}^{d} \rightarrow$ $\mathbb{R}$ can be approximated from below by $C^{\infty}$ convex functions, uniformly on bounded sets. And by Theorem 2 we get that every convex function $f: \mathbb{R}^{d} \rightarrow \mathbb{R}$ can be approximated from below by $C^{\infty}$ convex functions, uniformly on $\mathbb{R}^{d}$. Moreover, it is clear that strict (or strong) convexity, 
local Lipschitzness, and order are preserved by the combination of these techniques.

The case when $X=U$ is an open convex subset of $\mathbb{R}^{d}$ can be treated in a similar manner. We consider the open, bounded convex sets $B_{m}=\{x \in$ $U: \operatorname{dist}(x, \partial U)>1 / m,\|x\|<m\}$, so we have $\overline{B_{m}} \subset B_{m+1}$, $\operatorname{dist}\left(B_{m}, \partial U\right)>$ 0 and $U=\bigcup_{m=1}^{\infty} B_{m}$. By combining Theorem 2 and Proposition [3, it suffices to show that every Lipschitz, convex function $f: U \rightarrow \mathbb{R}$ can be approximated by $C^{\infty}$ convex functions, uniformly on $U$. This can be done as follows: set $L=\operatorname{Lip}(f)$ and consider

$$
g(x)=\inf \{f(y)+L\|x-y\|: y \in U\}, x \in \mathbb{R}^{d},
$$

which is a Lipschitz, convex extension of $f$ to all of $\mathbb{R}^{d}$, with $\operatorname{Lip}(f)=$ $\operatorname{Lip}(g)$. By using the above argument, $g$ can be approximated by $C^{\infty}$ convex functions, uniformly on $\mathbb{R}^{d}$. In particular, $f=g_{\left.\right|_{U}}$ can be approximated by such functions, uniformly on $U$. This proves Corollary 1 ,

Let us see how one can deduce Corollaries 2 and 3. As in the case of $\mathbb{R}^{d}$, the combination of Theorem 2 , Proposition 3 and Remark 1 reduces the problem to showing that every Lipschitz convex function $f: X \rightarrow \mathbb{R}$ (where $X$ stands for a Cartan-Hadamard manifold or a Banach space whose dual is locally uniformly convex) can be approximated by $C^{1}$ convex functions, uniformly on $X$. It is well known that this can be done via the inf convolution of $f$ with squared distances: the functions

$$
f_{\lambda}(x)=\inf \left\{f(y)+\frac{1}{2 \lambda} d(x, y)^{2}: y \in X\right\}
$$

are $C^{1}$, convex, Lipschitz (with the same constant as $f$ ), have the same minimizers as $f$, are strictly convex whenever $f$ is, and converge to $f$ as $\lambda \searrow 0$, uniformly on all of $X$. See 24] for a survey on the inf convolution operation in Banach spaces, and [2] for the Cartan-Hadamard case.

\section{Real anAlytic CONVEX approximations}

Let us now prove Theorem 1. As mentioned in the introduction, real analytic approximations of partitions of unity cannot be employed to glue local approximations into a uniform convex approximation of $f$ on all of $\mathbb{R}^{d}$.

A natural approach to this problem would be showing that every convex function can be approximated by $C^{2}$ strongly convex functions, and then using Whitney's theorem on $C^{2}$-fine approximation of functions by real analytic functions to conclude. However, not every convex function $f: \mathbb{R}^{d} \rightarrow \mathbb{R}$ can be approximated by strongly convex functions uniformly on $\mathbb{R}^{d}$. For instance, it is not possible to approximate a linear function by strongly convex functions.

We will show that, given a convex function $f: \mathbb{R}^{d} \rightarrow \mathbb{R}$, either we can reduce the problem of approximating $f$ by real analytic convex functions to some $\mathbb{R}^{k}$ with $k<d$, or else its graph is supported by a maximum of finitely many $(d+1)$-dimensional corners which besides approximates $f$ on a given 
bounded set (and which in turn we will manage to approximate by strongly convex functions).

Definition 4 (Supporting corners). We will say that a function $C: \mathbb{R}^{d} \rightarrow \mathbb{R}$ is a $k$-dimensional corner function on $\mathbb{R}^{d}$ if it is of the form

$$
C(x)=\max \left\{\ell_{1}+b_{1}, \ell_{2}+b_{2}, \ldots, \ell_{k}+b_{k}\right\},
$$

where the $\ell_{j}: \mathbb{R}^{d} \rightarrow \mathbb{R}$ are linear functions such that the functions $L_{j}$ : $\mathbb{R}^{d+1} \rightarrow \mathbb{R}$ defined by $L_{j}\left(x, x_{d+1}\right)=x_{d+1}-\ell_{j}(x), 1 \leq j \leq k$, are linearly independent, and the $b_{j} \in \mathbb{R}$. We will also say that a convex function $f$ : $U \subseteq \mathbb{R}^{d} \rightarrow \mathbb{R}$ is supported by $C$ at a point $x \in U$ provided we have $C \leq f$ on $U$ and $C(x)=f(x)$.

Lemma 2. If $C$ is a $(d+1)$-dimensional corner function on $\mathbb{R}^{d}$ then $C$ can be approximated by $C^{\infty}$ strongly convex functions, uniformly on $\mathbb{R}^{d}$.

Proof. We will need to use the following variation of the smooth maximum of Lemma 1; given $\varepsilon, r>0$, let $\beta_{\varepsilon, r}=|\cdot| * H_{r}+\varepsilon / 2$, where $H_{r}(x)=$ $\frac{1}{(4 \pi r)^{1 / 2}} \exp \left(-x^{2} / 4 r\right)$. We have $\beta_{\varepsilon, r}^{\prime \prime}(t)=2 e^{-t^{2} / 4 r} /(4 r \pi)^{1 / 2}>0$, so $\beta_{\varepsilon, r}$ is strongly convex and 1-Lipschitz, and as $r \rightarrow 0$ we have $\beta_{\varepsilon, r}(t) \rightarrow|t|+\varepsilon / 2$ uniformly on $t \in \mathbb{R}$, so we may find $r=r(\varepsilon)>0$ such that $|t| \leq \beta_{\varepsilon, r}(t) \leq$ $|t|+\varepsilon$ for all $t$. Put $\widetilde{\theta}_{\varepsilon}(t)=\beta_{\varepsilon, r(\varepsilon)}(t)$, and define $\widetilde{M}_{\varepsilon}: \mathbb{R}^{2} \rightarrow \mathbb{R}$ by

$$
\widetilde{M}_{\varepsilon}(x, y)=\frac{x+y+\widetilde{\theta}_{\varepsilon}(x-y)}{2} .
$$

It is clear that $\widetilde{M}_{\varepsilon}$ satisfies all the properties of Lemma 1 except for (iii).

Now let us prove our lemma. Up to an affine change of variables in $\mathbb{R}^{d+1}$, the problem is equivalent to showing that the function

$$
f(x)=\max \left\{0, x_{1}, x_{2}, \ldots, x_{d}\right\}
$$

can be uniformly approximated on $\mathbb{R}^{n}$ by $C^{\infty}$ strongly convex functions. We will show that this is possible by induction on $d$.

For $d=1$, the function $f(x)=\max \{x, 0\}$ is Lipschitz, so the convolutions $f_{\varepsilon}=f * H_{\varepsilon}$ are $C^{\infty}$, Lipschitz and converge to $f$, uniformly on $\mathbb{R}$, as $\varepsilon \searrow 0$. Besides, as one can easily compute,

$$
f_{\varepsilon}^{\prime \prime}(x)=\frac{1}{(4 \pi \varepsilon)^{1 / 2}} e^{-\frac{x^{2}}{4 \varepsilon}}>0,
$$

so the $f_{\varepsilon}$ are strongly convex.

Now, suppose that the function $f\left(x_{1}, \ldots, x_{k}\right)=\max \left\{0, x_{1}, \ldots, x_{k}\right\}$ can be uniformly approximated by $C^{\infty}$ smooth strongly convex functions on $\mathbb{R}^{k}$. Then, for a given $\varepsilon>0$ we can find $C^{\infty}$ strongly convex functions $g: \mathbb{R}^{k} \rightarrow \mathbb{R}$ and $\alpha: \mathbb{R} \rightarrow \mathbb{R}$ such that

$$
\begin{aligned}
& f(x) \leq g(x) \leq f(x)+\varepsilon \text { for all } x \in \mathbb{R}^{k}, \quad \text { and } \\
& \max \{t, 0\} \leq \alpha(t) \leq \max \{t, 0\}+\varepsilon \text { for all } t \in \mathbb{R} .
\end{aligned}
$$


Given the function

$$
F\left(x_{1} \ldots, x_{k}, x_{k+1}\right)=\max \left\{0, x_{1}, \ldots, x_{k+1}\right\}=\max \left\{x_{k+1}, f\left(x_{1}, \ldots, x_{k}\right)\right\},
$$

let us define $G: \mathbb{R}^{k+1} \rightarrow \mathbb{R}$ by

$$
G\left(x_{1}, \ldots, x_{k+1}\right)=\widetilde{M}_{\varepsilon}\left(g\left(x_{1}, \ldots, x_{k}\right), \alpha\left(x_{k+1}\right)\right) .
$$

We have $G \in C^{\infty}\left(\mathbb{R}^{k+1}\right)$, and $F(x) \leq G(x) \leq F(x)+2 \varepsilon$ for all $x \in \mathbb{R}^{k+1}$, so in order to conclude the proof we only have to see that $G$ is strongly convex. Given $x, v \in \mathbb{R}^{k+1}$ with $v \neq 0$, it is enough to check that the function

$$
h(t):=G(x+t v)=\widetilde{M}_{\varepsilon}(\beta(t), \gamma(t)),
$$

where $\beta(t)=g\left(x_{1}+t v_{1}, \ldots, x_{k}+t v_{k}\right)$ and $\gamma(t)=\alpha\left(x_{k+1}+t v_{k+1}\right)$, satisfies $h^{\prime \prime}(t)>0$. If $v_{k+1} \neq 0$ and $\left(v_{1}, \ldots, v_{k}\right) \neq 0$ then, since $g$ is strongly convex on $\mathbb{R}^{k}$ and $\alpha$ is strongly convex on $\mathbb{R}$, we have $\beta^{\prime \prime}(t)>0$ and $\gamma^{\prime \prime}(t)>0$, so exactly as in the proof of (9) of Proposition 2 we also get $h^{\prime \prime}(t)>0$. On the other hand, if for instance we have $v_{k+1}=0$ then $\beta^{\prime \prime}(t)>0$ and $\gamma^{\prime}(t)=\gamma^{\prime \prime}(t)=0$, so

$\frac{d^{2}}{d t^{2}} \widetilde{M}_{\varepsilon}(\beta(t), \gamma(t))=\frac{\left(1+\theta_{\varepsilon}^{\prime}(\beta(t)-\gamma(t))\right) \beta^{\prime \prime}(t)+\theta_{\varepsilon}^{\prime \prime}(\beta(t)-\gamma(t))(\beta(t)-\gamma(t))^{2}}{2}>0$,

because $\left|\widetilde{\theta}_{\varepsilon}^{\prime}\right| \leq 1, \widetilde{\theta}_{\varepsilon}^{\prime \prime}>0$, and $\widetilde{\theta}_{\varepsilon}^{\prime}(0)=0$. Similarly one checks that $\frac{d^{2}}{d t^{2}} \widetilde{M}_{\varepsilon}(\beta(t), \gamma(t))>0$ in the case when $\left(v_{1}, \ldots, v_{k}\right)=0 \neq v_{k+1}$.

Lemma 3. Let $U \subseteq \mathbb{R}^{n}$ be open and convex, $f: U \rightarrow \mathbb{R}$ be a $C^{p}$ convex function, and $x_{0} \in U$. Assume that $f$ is not supported at $x_{0}$ by any $(n+1)$ dimensional corner function. Then there exist $k<n$, a linear projection $P: \mathbb{R}^{n} \rightarrow \mathbb{R}^{k}$, a $C^{p}$ convex function $c: P(U) \subseteq \mathbb{R}^{k} \rightarrow \mathbb{R}$, and a linear function $\ell: \mathbb{R}^{n} \rightarrow \mathbb{R}$ such that $f=c \circ P+\ell$.

Proof. If $f$ is affine the result is obvious. If $f$ is not affine then there exists $y_{0} \in U$ with $f^{\prime}\left(x_{0}\right) \neq f^{\prime}\left(y_{0}\right)$. It is clear that $L_{1}\left(x, x_{n+1}\right)=x_{n+1}-f^{\prime}\left(x_{0}\right)(x)$ and $L_{2}\left(x, x_{n+1}\right)=x_{n+1}-f^{\prime}\left(y_{0}\right)(x)$ are two linearly independent linear functions on $\mathbb{R}^{n+1}$, hence $f$ is supported at $x_{0}$ by the two-dimensional corner $x \mapsto \max \left\{f\left(x_{0}\right)+f^{\prime}\left(x_{0}\right)\left(x-x_{0}\right), f\left(y_{0}\right)+f^{\prime}\left(y_{0}\right)\left(x-y_{0}\right)\right\}$. Let us define $m$ as the greatest integer number so that $f$ is supported at $x_{0}$ by an $m$ dimensional corner. By assumption we have $2 \leq m<n+1$. Define $k=m-1$. There exist $\ell_{1}, \ldots, \ell_{k+1} \in\left(\mathbb{R}^{n}\right)^{*}$ with $L_{j}\left(x, x_{n+1}\right)=x_{n+1}-\ell_{j}(x)$, $j=1, \ldots, k+1$, linearly independent in $\left(\mathbb{R}^{n+1}\right)^{*}$, and $b_{1}, \ldots, b_{k+1} \in \mathbb{R}$, so that $C=\max _{1 \leq j \leq k+1}\left\{\ell_{j}+b_{j}\right\}$ supports $f$ at $x_{0}$.

Observe that the $\left\{L_{j}-L_{1}\right\}_{j=2}^{k+1}$ are linearly independent in $\left(\mathbb{R}^{n+1}\right)^{*}$, hence so are the $\left\{\ell_{j}-\ell_{1}\right\}_{j=2}^{k+1}$ in $\left(\mathbb{R}^{n}\right)^{*}$, and therefore $\bigcap_{j=2}^{k+1} \operatorname{Ker}\left(\ell_{j}-\ell_{1}\right)$ has dimension $n-k$. Then we can find linearly independent vectors $w_{1}, \ldots, w_{n-k}$ such that $\bigcap_{j=2}^{k+1} \operatorname{Ker}\left(\ell_{j}-\ell_{1}\right)=\operatorname{span}\left\{w_{1}, \ldots, w_{n-k}\right\}$.

Now, given any $y \in U$, if $\left.\frac{d}{d t}\left(f-\ell_{1}\right)\left(y+t w_{q}\right)\right|_{t=t_{0}} \neq 0$ for some $t_{0}$ then $f^{\prime}\left(y+t_{0} w_{q}\right)-\ell_{1}$ is linearly independent with $\left\{\ell_{j}-\ell_{1}\right\}_{j=2}^{k+1}$, which implies that 
$\left(x, x_{n+1}\right) \mapsto x_{n+1}-f^{\prime}\left(y+t_{0} w_{q}\right)$ is linearly independent with $L_{1}, \ldots, L_{k+1}$, and therefore the function

$x \mapsto \max \left\{\ell_{1}(x)+b_{1}, \ldots, \ell_{k+1}(x)+b_{k+1}, f^{\prime}\left(y+t_{0} w_{q}\right)\left(x-y-t_{0} w_{q}\right)+f\left(y+t_{0} w_{q}\right)\right\}$

is a $(k+2)$-dimensional corner supporting $f$ at $x_{0}$, which contradicts the choice of $m$. Therefore we must have

$\frac{d}{d t}\left(f-\ell_{1}\right)\left(y+t w_{q}\right)=0$ for all $y \in U, t \in \mathbb{R}$ with $y+t w_{q} \in U, q=1, \ldots, n-k$.

This implies that

$$
\left(f-\ell_{1}\right)\left(y+\sum_{j=1}^{n-k} t_{j} w_{j}\right)=\left(f-\ell_{1}\right)(y)
$$

if $y \in U$ and $y+\sum_{j=1}^{n-k} t_{j} w_{j} \in U$. Let $Q$ be the orthogonal projection of $\mathbb{R}^{n}$ onto the subspace $E:=\operatorname{span}\left\{w_{1}, \ldots, w_{n-k}\right\}^{\perp}$. For each $z \in Q(U)$ we may define

$$
\widetilde{c}(z)=\left(f-\ell_{1}\right)\left(z+\sum_{j=1}^{n-k} t_{j} w_{j}\right)
$$

if $z+\sum_{j=1}^{n-k} t_{j} w_{j} \in U$ for some $t_{1}, \ldots, t_{n-k}$. It is clear that $\widetilde{c}: Q(U) \rightarrow \mathbb{R}$ is well defined, convex and $C^{p}$, and satisfies

$$
f-\ell_{1}=\widetilde{c} \circ Q .
$$

Then, by taking a linear isomorphism $T: E \rightarrow \mathbb{R}^{k}$ and setting $P=T Q$, we have that $f=c \circ P+\ell_{1}$, where $c=\widetilde{c} \circ T^{-1}$ is defined on $P(U)$.

Now we can prove Theorem 1. We already know that a convex function $f: U \subseteq \mathbb{R} \rightarrow \mathbb{R}$ can be uniformly approximated from below by $C^{1}$ functions, so we may assume that $f \in C^{1}(U)$. We will proceed by induction on $d$, the dimension of $\mathbb{R}^{d}$.

For $d=1$ the result can be proved as follows. Either $f: U \rightarrow \mathbb{R}$ is affine (in which case we are done) or $f$ can be supported by a 2-dimensional corner at every point $x \in U$. In the latter case, let us consider a compact interval $I \subset U$. Given $\varepsilon>0$, since $f$ is convex and Lipschitz on $I$ we can find finitely many affine functions $h_{1}, \ldots, h_{m}: \mathbb{R} \rightarrow \mathbb{R}$ such that each $h_{j}$ supports $f-\varepsilon$ at some point $x_{j} \in I$ and $f-2 \varepsilon \leq \max \left\{h_{1}, \ldots, h_{m}\right\}$ on $I$. By convexity we also have $\max \left\{h_{1}, \ldots, h_{m}\right\} \leq f-\varepsilon$ on all of $U$. For each $x_{j}$ we may find a 2-dimensional corner $C_{j}$ which supports $f-\varepsilon$ at $x_{j}$. Since $f$ is differentiable and convex we have $h_{j}=C_{j}$ on a neighbourhood of $x_{j}$ and, by convexity, also $h_{j} \leq C_{j} \leq f-\varepsilon$ and $\max \left\{C_{1}, \ldots, C_{m}\right\} \leq f-\varepsilon$ on $U$. And we also have $f-2 \varepsilon \leq \max \left\{h_{1}, \ldots, h_{m}\right\} \leq \max \left\{C_{1}, \ldots, C_{m}\right\} \leq f-\varepsilon$ on $I$. Now apply Lemma 2 to find $C^{\infty}$ strongly convex functions $g_{1}, \ldots, g_{m}: \mathbb{R} \rightarrow \mathbb{R}$ such that $C_{j} \leq g_{j} \leq C_{j}+\varepsilon^{\prime}$, where $\varepsilon^{\prime}:=\varepsilon / 2 m$, and define $g: \mathbb{R} \rightarrow \mathbb{R}$ by

$$
g=M_{\varepsilon^{\prime}}\left(g_{1}, M_{\varepsilon^{\prime}}\left(g_{2}, M_{\varepsilon^{\prime}}\left(g_{3}, \ldots, M_{\varepsilon^{\prime}}\left(g_{m-1}, g_{m}\right)\right) \ldots\right)\right)
$$


(for instance, if $m=3$ then $g=M_{\varepsilon^{\prime}}\left(g_{1}, M_{\varepsilon^{\prime}}\left(g_{2}, g_{3}\right)\right)$ ). By Proposition 2 we have that $g \in C^{\infty}(\mathbb{R})$ is strongly convex,

$$
\max \left\{C_{1}, \ldots, C_{m}\right\} \leq g \leq \max \left\{C_{1}, \ldots, C_{m}\right\}+m \varepsilon^{\prime} \leq f-\frac{\varepsilon}{2} \quad \text { on } \quad U,
$$

and

$$
f-2 \varepsilon \leq \max \left\{C_{1}, \ldots, C_{m}\right\} \leq g \text { on } I .
$$

Therefore $f: U \subseteq \mathbb{R} \rightarrow \mathbb{R}$ can be approximated from below by $C^{\infty}$ strongly convex functions, uniformly on compact subintervals of $U$. By Theorem 2 and Remark 1 we conclude that, given $\varepsilon>0$ we may find a $C^{\infty}$ strongly convex function $h$ such that $f-2 \varepsilon \leq h \leq f-\varepsilon$ on $U$.

Finally, set $\eta(x)=\frac{1}{2} \min \left\{h^{\prime \prime}(x), \varepsilon\right\}$ for every $x \in U$. The function $\eta: U \rightarrow(0, \infty)$ is continuous, so we can apply Whitney's theorem on $C^{2}$ fine approximation of $C^{2}$ functions by real analytic functions to find a real analytic function $g: U \rightarrow \mathbb{R}$ such that

$$
\max \left\{|h-g|,\left|h^{\prime}-g^{\prime}\right|,\left|h^{\prime \prime}-g^{\prime \prime}\right|\right\} \leq \eta .
$$

This implies that $f-3 \varepsilon \leq g \leq f$ and $g^{\prime \prime} \geq \frac{1}{2} h^{\prime \prime}>0$, so $g$ is strongly convex as well.

Now assume the result is true in $\mathbb{R}, \mathbb{R}^{2}, \ldots, \mathbb{R}^{d}$, and let us see that then it is also true in $\mathbb{R}^{d+1}$. If there is some $x_{0} \in U$ such that $f: U \subseteq \mathbb{R}^{d+1} \rightarrow \mathbb{R}$ is not supported at $x_{0}$ by any $(d+2)$-dimensional corner function then, according to Lemma 3, we can find $k \leq d$, a linear projection $P: \mathbb{R}^{d+1} \rightarrow \mathbb{R}^{k}$, a linear function $\ell: \mathbb{R}^{d+1} \rightarrow \mathbb{R}$, and a $C^{\infty}$ convex function $c: P(U) \rightarrow \mathbb{R}$ such that $f=c \circ P+\ell$. By assumption there exists a real analytic convex function $h: P(U) \subseteq \mathbb{R}^{k} \rightarrow \mathbb{R}$ so that $c-\varepsilon \leq h \leq c$. Then the function $g=h \circ P+\ell$ is real analytic, convex (though never strongly convex), and satisfies $f-\varepsilon \leq g \leq f$.

If there is no such $x_{0}$ then one can repeat exactly the same argument as in the case $d=1$, just replacing 2-dimensional corners with $(d+2)$-dimensional corners, the interval $I$ with a compact convex body $K \subset U$, and $\eta$ with

$$
\eta(x)=\frac{1}{2} \min \left\{\varepsilon, \min \left\{D^{2} h(x)(v)^{2}: v \in \mathbb{R}^{d+1},\|v\|=1\right\}\right\},
$$

in order to conclude that there exists a real analytic strongly convex $g: U \rightarrow$ $\mathbb{R}$ such that $f-\varepsilon \leq g \leq f$ on $U$.

Incidentally, the above argument also shows Proposition 1 in the case when $f$ is $C^{1}$. In the general case of a nonsmooth convex function one just needs to take two more facts into account. First, Lemma 3 holds for nonsmooth convex functions (to see this, use the fact that if the range of the subdifferential of a convex function is contained in $\{0\}$ then the function is constant, see for instance [6, Chapter 1, Corollary 2.7], and apply this to the function $\left.\left(t_{1}, \ldots, t_{d-k}\right) \mapsto\left(f-\ell_{1}\right)\left(y+\sum_{j=1}^{d-k} t_{j} w_{j}\right)\right)$. Second, in the above proof one can use Rademacher's theorem and uniform continuity of $f$ to see that the $x_{j}$ can be assumed to be points of differentiability of $f$. 


\section{5. $C^{0}$-Fine APPROXIMATION OF GENERAL CONVEX FUNCTIONS IS IMPOSSIBLE: THREE COUNTEREXAMPLES}

We start to discuss the possibility of approximating a convex function $f: \mathbb{R}^{d} \rightarrow \mathbb{R}$ by smooth convex functions in the $C^{0}$-fine topology. We will see that there is quite a big difference between the cases $d=1$ and $d \geq 2$.

In the case $n=1$ we will show that every convex function $f: \mathbb{R} \rightarrow \mathbb{R}$ can be approximated by convex real analytic functions in this topology. However, this approximation cannot be performed from below:

Example 1. Let $f: \mathbb{R} \rightarrow \mathbb{R}$ be defined by $f(x)=|x|$. For every $C^{1}$ convex function $g: \mathbb{R} \rightarrow \mathbb{R}$ such that $g(0) \leq 0$ we have

$$
\liminf _{|x| \rightarrow \infty}|f(x)-g(x)|>0 .
$$

In particular, if $\varepsilon: \mathbb{R} \rightarrow(0, \infty)$ is continuous and satisfies $\lim _{|x| \rightarrow \infty} \varepsilon(x)=0$ then there is no $C^{1}$ convex function $g: \mathbb{R} \rightarrow \mathbb{R}$ such that $|x|-\varepsilon(x) \leq g(x) \leq$ $|x|$.

In two or more dimensions the situation gets much worse: $C^{0}$-fine approximation of convex functions by $C^{1}$ convex functions is no longer possible in general.

Example 2. For $d \geq 2$, let $f: \mathbb{R}^{d} \rightarrow \mathbb{R}$ be defined by $f\left(x_{1}, \ldots, x_{d}\right)=\left|x_{1}\right|$, and let $\varepsilon: \mathbb{R}^{d} \rightarrow(0, \infty)$ be continuous with $\lim _{|x| \rightarrow \infty} \varepsilon(x)=0$. Then there is no $C^{1}$ convex function $g: \mathbb{R}^{d} \rightarrow \mathbb{R}$ such that $|f-g| \leq \varepsilon$.

Our last example shows that when $U \neq \mathbb{R}^{d}, d \geq 2$, it is possible to construct convex functions $f: U \rightarrow \mathbb{R}$ which cannot be approximated by smoother convex functions in the $C^{0}$-fine topology, and which are not of the form $f=c \circ P+\ell$ (where $P: \mathbb{R}^{d} \rightarrow \mathbb{R}^{k}, k<d, c: P(U) \rightarrow \mathbb{R}$ convex and $\ell$ linear).

Example 3. Let $\varphi$ be a $C^{p}$ strongly convex function on $\mathbb{R}$ which is not $C^{p+1}$ on any neighbourhood of 0 , and let $\psi: \mathbb{R} \rightarrow \mathbb{R}$ be a $C^{\infty}$ function such that $\psi=0$ on $[-\varepsilon(1+\varepsilon), \varepsilon(1+\varepsilon)]$, and $\min \left\{\psi, \psi^{\prime \prime}\right\}>0$ on $\mathbb{R} \backslash[-\varepsilon(1+\varepsilon), \varepsilon(1+\varepsilon)]$. Let $U=(-1,1) \times(-1,1) \subset \mathbb{R}^{2}, \varepsilon \in(0,1)$, and define $f: U \rightarrow \mathbb{R}$ by

$$
f(x, y)=\varphi(x)+\psi(x+\varepsilon y)+\psi(x-\varepsilon y) .
$$

Notice that $f$ is strongly convex outside the set $C_{\varepsilon}=\{(x, y) \in U:-\varepsilon(1+$ $\varepsilon) \leq x+\varepsilon y \leq \varepsilon(1+\varepsilon),-\varepsilon(1+\varepsilon) \leq x-\varepsilon y \leq \varepsilon(1+\varepsilon)\}$, and the measure of $C_{\varepsilon}$ is less than $2 \varepsilon(1+\varepsilon)$. It is not difficult to see that if $\varepsilon: \mathbb{R}^{2} \rightarrow[0, \infty)$ is a $C^{1}$ function with $\varepsilon^{-1}(0)=\mathbb{R}^{2} \backslash U$ then there is no convex function $g \in C^{p+1}(U)$ such that $|f-g| \leq \varepsilon$ on $U$.

6. $C^{0}$-Fine APPROXIMATION OF PROPERLy CONVEX FUNCTIONS. A GLUING TECHNIQUE FOR PROPER FUNCTIONS.

We start proving Theorem 3. We may write $f=\ell+c$, where $\ell$ is linear and $c$ is convex and proper. Since addition of linear functions preserves 
convexity, smoothness, and the kind of approximation we are dealing with, in order to prove our result we may assume that $\ell=0$, and in particular that $f: U \rightarrow[a, b)$ is proper and attains a minimum at some point $x_{0} \in U$ with $f\left(x_{0}\right)=a$.

For every $n \in \mathbb{N}$ let us define

$$
B_{n}=f^{-1}\left[a, \beta_{n}\right),
$$

where $\left(\beta_{n}\right)$ is a strictly increasing sequence of real numbers converging to $b$. Each $\overline{B_{n}}=f^{-1}\left[a, \beta_{n}\right]$ is a compact convex body with interior $B_{n}$, and we have

$$
U=\bigcup_{n=1}^{\infty} B_{n} \text {, and } \overline{B_{n}} \subset B_{n+1} \text { for all } n \text {. }
$$

We also have

$$
\widetilde{\alpha}:=\inf _{U \backslash B_{1}} f-f\left(x_{0}\right)=\beta_{1}-a>0 .
$$

For each $v \in \mathbb{R}^{d}$ with $\|v\|=1$ let us consider the function $\psi(t)=\psi_{x_{0}, v}(t)=$ $f(x+t v)$. There are unique numbers $\tau_{n}^{ \pm}$such that $\tau_{n+1}^{-}<\tau_{n}^{-}<\ldots<\tau_{1}^{-}<$ $0<\tau_{1}^{+}<\ldots<\tau_{n}^{+}<\tau_{n+1}^{+}$and $x_{0}+\tau_{n}^{ \pm} v \in \partial B_{n}$ for all $n$. By convexity of $\psi$, for every $\eta_{n}^{ \pm} \in \partial \psi\left(\tau_{n}^{ \pm}\right)$we have $\eta_{n+1}^{-} \leq \eta_{n}^{-} \leq \ldots \leq \eta_{1}^{-} \leq 0 \leq \eta_{1}^{+} \leq \ldots \leq \eta_{n}^{+} \leq$ $\eta_{n+1}^{+}$. Then, for every $\zeta_{n}^{ \pm} \in \partial f\left(x_{0}+\tau_{n}^{ \pm} v\right)$ we have that $\eta_{n}=\zeta_{n}^{ \pm}(v) \in \partial \psi\left(\tau_{n}^{ \pm}\right)$ and therefore

$$
\left\|\zeta_{n}^{+}\right\| \geq \zeta_{n}^{+}(v) \geq \eta_{1}^{+}(v) \geq \frac{\psi\left(\tau_{1}^{+}\right)-\psi(0)}{\tau_{1}^{+}} \geq \frac{\widetilde{\alpha}}{\operatorname{diam}\left(B_{1}\right)}:=\alpha>0 .
$$

Since $v$ is an arbitrary unit vector, this shows in particular that

$$
\inf \left\{\|\zeta\|: \zeta \in \partial f(y), y \in \partial B_{n}, n \in \mathbb{N}\right\} \geq \alpha>0 .
$$

(A similar argument shows that if $v$ is a unit vector transversal to $\partial B_{n}$ at $y \in \partial B_{n}$ such that $y+t v \in B_{n}$ for $t>0$ sufficiently small, then the function $t \mapsto f(y+t v)$ is strictly decreasing on an interval $(-\delta, \delta)$, for some $\delta>0$ sufficiently small.)

Next, associated to each $B_{n}$ we define a function $f_{n}: \mathbb{R}^{d} \rightarrow \mathbb{R}$ by

$$
f_{n}(x)=\inf \left\{f(y)+L_{n+2}|x-y|: y \in \overline{B_{n+2}}\right\},
$$

where $L_{n+2}$ is the Lipschitz constant of $f_{\left.\right|_{B_{n+2}}}$.

Claim 4. The $f_{n}$ are Lipschitz convex functions on $\mathbb{R}^{d}$ such that

$$
\begin{gathered}
f_{n} \leq f_{n+1} \text { on } \mathbb{R}^{d}, \\
f_{n}=f \text { on } B_{n+2} .
\end{gathered}
$$

Moreover, $\lim _{|x| \rightarrow \infty} f_{n}(x)=\infty$, and $f_{n}$ can be supported by a $(d+1)$ dimensional corner function at every point $x \in \mathbb{R}^{d}$. 
Proof. It is a well known fact that $f_{n}$ is an $L_{n+2}$-Lipschitz convex extension of $f_{\left.\right|_{B_{n+2}}}$ to all of $\mathbb{R}^{d}$, and it is easy to check that $f_{n} \leq f_{n+1}$ for all $n$. Let us check that $\lim _{|x| \rightarrow \infty} f_{n}(x)=\infty$. For every $y \in \partial B_{1}$, there exists a unique unit vector $v=v_{y}$ such that the ray $x_{0}+t v, t>0$ intersects $\partial B_{1}$ at a unique time $\tau_{y}$. Necessarily, $\tau_{y} \leq \operatorname{diam}\left(B_{1}\right)$. According to (3) above, we have $\zeta_{y}(v) \geq \alpha$. Write $x=x_{t, v}=x_{0}+t v$. By convexity of $f_{n}$ we have

$$
\begin{aligned}
& f_{n}(x)=f_{n}\left(x_{0}+t v\right) \geq f_{n}(y)+\left(t-\tau_{y}\right) \zeta_{y}(v)=f(y)+\left(t-\tau_{y}\right) \zeta_{y}(v) \geq \\
& f(y)+\alpha\left(t-\tau_{y}\right) \geq a+\alpha\left(\left|x-x_{0}\right|-\operatorname{diam}\left(B_{1}\right)\right),
\end{aligned}
$$

hence $f_{n}(x) \rightarrow \infty$ as $|x| \rightarrow \infty$. Finally, according to Lemma 3, if $f_{n}$ could not be supported by a $(d+1)$-dimensional corner function at each $x \in \mathbb{R}^{d}$ then we would have $f_{n}=c_{n} \circ P_{n}+\ell_{n}$ for some linear projection $P_{n}: \mathbb{R}^{d} \rightarrow \mathbb{R}^{k_{n}}, k_{n}<$ $d, c_{n}: P_{n}(U) \rightarrow \mathbb{R}$ convex, and $\ell_{n}: \mathbb{R}^{d} \rightarrow \mathbb{R}$ linear. But this is impossible, since for $y \in \operatorname{Ker} P_{n} \backslash\{0\}$ we have $c_{n}\left(P_{n}(t y)\right)+\ell_{n}(t y)=c_{n}(0)+t \ell_{n}(y)$, which does not go to $\infty$ as $|t| \rightarrow \infty$.

Now, given a continuous function $\varepsilon: U \rightarrow(0, \infty)$, define

$$
\varepsilon_{n}=\frac{1}{6} \min \left\{\varepsilon(x): x \in \overline{B_{n+1}}\right\} .
$$

Associated to each $B_{n}$, and for every number $r_{n} \in(0,1)$ let us also define functions $\widetilde{f}_{n}=\widetilde{f}_{n, r_{n}}$ by

$$
\tilde{f}_{n}(x)=\left(1-r_{n}\right)\left(f_{n}(x)-\beta_{n}\right)+\beta_{n} .
$$

Claim 5. The functions $\widetilde{f}_{n}=\widetilde{f}_{n, r_{n}}$ are convex and Lipschitz, and the $r_{n}$ can be chosen small enough so as to have

$$
\begin{gathered}
f_{n}<\tilde{f}_{n}<f_{n}+\varepsilon_{n} \text { on } B_{n}, \\
f_{n}=\widetilde{f}_{n} \text { on } \partial B_{n}, \\
\widetilde{f}_{n}<f_{n} \text { on } \mathbb{R}^{d} \backslash \overline{B_{n}}, \text { and } \\
f_{n}-\varepsilon_{n}<\widetilde{f}_{n}<f_{n} \text { on } B_{n+1} \backslash \overline{B_{n}} .
\end{gathered}
$$

Moreover, $\lim _{|x| \rightarrow \infty} \widetilde{f}_{n}(x)=\infty$, and $\widetilde{f}_{n}$ can be supported by a $(d+1)$ dimensional corner function at every point $x \in \mathbb{R}^{d}$.

Proof. For $\varepsilon \in(0,1)$, denote $f_{n, \varepsilon}=(1-\varepsilon)\left(f_{n}(x)-\beta_{n}\right)+\beta_{n}$. It is clear that $f_{n}<f_{n, \varepsilon}$ on $B_{n}$ and $f_{n, \varepsilon}<f_{n}$ on $\mathbb{R}^{d} \backslash \overline{B_{n}}$. Since $\lim _{\varepsilon \rightarrow 0^{+}} \widetilde{f}_{n, \varepsilon}=f_{n}$ uniformly on compact subsets of $\mathbb{R}^{d}$, we can find $\varepsilon=r_{n} \in(0,1)$ such that all the inequalities in the statement hold true. On the other hand, by Claim 4 we get that $\lim _{|x| \rightarrow \infty} \widetilde{f}_{n}(x)=\infty$, hence, by the same argument as in the proof of Claim $4, \widetilde{f}_{n}$ must also be supported by $(d+1)$-dimensional corners at each point $x \in \mathbb{R}^{d}$. 
Claim 6. We can find numbers $\left\{r_{n}\right\} \subset(0,1)$ with $r_{n+1}<r_{n}$ for all $n$, $r_{n} \searrow 0$ and as in the preceding claim, open convex sets $A_{n}, C_{n}$, an open neighbourhood $\mathcal{N}_{n}$ of $\partial A_{n}$, and numbers $s_{n}>0$ such that

$$
\overline{A_{n}} \subset B_{n} \subset \overline{B_{n}} \subset C_{n} \subset \overline{C_{n}} \subset A_{n+1},
$$

and the function $\widetilde{f}_{n}=\widetilde{f}_{n, r_{n}}$ satisfies

$$
\begin{aligned}
& \widetilde{f}_{n}+s_{n} \leq \min \left\{f_{n}, \widetilde{f}_{n+1}\right\} \text { on } U \backslash C_{n}, \\
& \widetilde{f}_{n}-\varepsilon_{n} \leq f_{n} \leq \widetilde{f}_{n}+\varepsilon_{n} \text { on } C_{n}, \quad \text { and } \\
& \widetilde{f}_{n} \geq \widetilde{f}_{n+1}+s_{n} \geq f+s_{n} \text { on } A_{n} \cup \mathcal{N}_{n} .
\end{aligned}
$$

Proof. This follows from Claim 5 and a standard compactness argument.

Now we are ready to construct a $C^{\infty}$ strongly convex function $g: U \rightarrow \mathbb{R}$ such that $|f(x)-g(x)| \leq \varepsilon(x)$ for every $x \in U$. We will do this by means of an inductive process. We start considering the function $\tilde{f}_{1}$. According to the proof of Theorem 1 , because $\widetilde{f}_{1}$ can be supported by $(d+1)$-dimensional corner functions at every point, we can find a strongly convex function $\varphi_{1} \in$ $C^{\infty}(U)$ such that $\tilde{f}_{1}-\varepsilon_{1}^{\prime} \leq \varphi_{1} \leq \widetilde{f}_{1}$ on $U$, where $\varepsilon_{1}^{\prime}:=\frac{1}{2} \min \left\{\varepsilon_{1}, s_{1}\right\}$. Set $g_{1}=\varphi_{1}$.

Claim 7. We have $\left|g_{1}-f\right| \leq 2 \varepsilon_{1}$ on $C_{1}$.

Proof. On $C_{1}$, on the one hand, $g_{1} \leq \widetilde{f}_{1} \leq f_{1}+\varepsilon_{1} \leq f+2 \varepsilon_{1}$, and on the other hand, $g_{1} \geq \tilde{f}_{1}-\varepsilon_{1}^{\prime} \geq f_{1}-\varepsilon_{1}-\varepsilon_{1}^{\prime} \geq f-2 \varepsilon_{1}$.

Next, consider $\widetilde{f}_{2}$, and set

$$
\delta_{2}:=\frac{s_{1}}{2}, \text { and } \varepsilon_{2}^{\prime}:=\frac{1}{2} \min \left\{\varepsilon_{2}, s_{2}, \varepsilon_{1}^{\prime}\right\} .
$$

As before, we can find a strongly convex function $\varphi_{2} \in C^{\infty}(U)$ such that $\widetilde{f}_{2}-\varepsilon_{2}^{\prime} \leq \varphi_{2} \leq \widetilde{f}_{2}$ on $U$. Define $g_{2}: U \rightarrow \mathbb{R}$ by

$$
g_{2}(x)= \begin{cases}g_{1}(x), & \text { if } x \in A_{1} \\ M_{\delta_{2}}\left(g_{1}(x), \varphi_{2}(x)\right), & \text { if } x \in U \backslash A_{1},\end{cases}
$$

where $M_{\delta_{2}}$ is the corresponding smooth maximum defined in Lemma 1 .

Claim 8. The function $g_{2}$ is well defined, strongly convex, $C^{\infty}$, and satisfies

$$
\begin{gathered}
g_{2}=g_{1} \text { on } A_{1}, \\
\left|g_{2}-f\right| \leq 3 \varepsilon_{1} \text { on } C_{1}, \\
g_{2}=\varphi_{2} \text { on } U \backslash C_{1}, \text { and } \\
\left|g_{2}-f\right| \leq 2 \varepsilon_{2} \text { on } C_{2} \backslash C_{1} .
\end{gathered}
$$


Proof. Let $x \in \mathcal{N}_{1}$, then we have

$g_{1}(x)=\varphi_{1}(x) \geq \widetilde{f}_{1}(x)-\varepsilon_{1}^{\prime} \geq f(x)+s_{1}-s_{1} / 2=f_{2}(x)+\delta_{2} \geq \varphi_{2}(x)+\delta_{2}$,

hence $M_{\delta_{2}}\left(g_{1}(x), \varphi_{2}(x)\right)=g_{1}(x)$. Using Proposition 2 this implies that $g_{2}$ is well defined, convex and $C^{\infty}$. By definition $g_{2}=g_{1}$ on $A_{1}$. Let us see that $g_{2}=\varphi_{2}$ on $U \backslash C_{1}$. If $x \in U \backslash C_{1}$,

$$
g_{1}(x)=\varphi_{1}(x) \leq \widetilde{f}_{1}(x) \leq \widetilde{f}_{2}(x)-s_{1} \leq \varphi_{2}(x)+\varepsilon_{2}^{\prime}-s_{1} \leq \varphi_{2}(x)-\delta_{2}
$$

hence $M_{\delta_{2}}\left(g_{1}(x), \varphi_{2}(x)\right)=\varphi_{2}(x)$.

Let us now see that $\left|g_{2}-f\right| \leq 3 \varepsilon_{1}$ on $C_{1}$. On the one hand we have, for every $x \in C_{1}$,

$$
g_{2}(x) \leq \max \left\{g_{1}(x), \varphi_{2}(x)\right\}+\delta_{2} \leq \max \left\{f+2 \varepsilon_{1}, f+\varepsilon_{2}\right\}+\delta_{2} \leq f+3 \varepsilon_{1},
$$

and on the other hand $g_{2}(x) \geq \max \left\{g_{1}(x), \varphi_{2}(x)\right\} \geq f(x)-2 \varepsilon_{1}$.

Finally, on $C_{2} \backslash C_{1}$ we have $g_{2}=\varphi_{2}$ so, as in Claim 7, we get $\left|g_{2}-f\right| \leq 2 \varepsilon_{2}$ on $C_{2} \backslash C_{1}$.

Now consider $\widetilde{f_{3}}$ and put

$$
\delta_{3}:=\frac{s_{2}}{2}, \text { and } \varepsilon_{3}^{\prime}:=\frac{1}{2} \min \left\{\varepsilon_{3}, s_{3}, \varepsilon_{2}^{\prime}\right\}
$$

find a strongly convex function $\varphi_{3} \in C^{\infty}(U)$ such that $f_{3}-\varepsilon_{3}^{\prime} \leq \varphi_{3} \leq f_{3}$ on $U$, and define

$$
g_{3}(x)= \begin{cases}g_{2}(x), & \text { if } x \in A_{2} \\ M_{\delta_{3}}\left(g_{2}(x), \varphi_{3}(x)\right), & \text { if } x \in U \backslash A_{2} .\end{cases}
$$

As in the preceding claim, it is not difficult to check that $g_{3}$ is well defined, strongly convex, $C^{\infty}$, and satisfies

$$
\begin{gathered}
g_{3}=g_{2} \text { on } A_{2}, \\
\left|g_{3}-f\right| \leq 3 \varepsilon_{2} \text { on } C_{2} \backslash C_{1}, \\
g_{3}=\varphi_{2} \text { on } U \backslash C_{2}, \text { and } \\
\left|g_{3}-f\right| \leq 2 \varepsilon_{3} \text { on } C_{3} \backslash C_{2} .
\end{gathered}
$$

By continuing the inductive process in this manner one can construct a sequence of strongly convex functions $g_{n} \in C^{\infty}(U)$ such that

$$
\begin{gathered}
g_{n+1}=g_{n} \text { on } A_{n} \\
\left|g_{n+1}-f\right| \leq 3 \varepsilon_{n} \text { on } C_{n} \backslash C_{n-1}, \\
\left|g_{n+1}-f\right| \leq 2 \varepsilon_{n+1} \text { on } C_{n+1} \backslash C_{n},
\end{gathered}
$$

and with $\left|g_{1}-f\right| \leq 2 \varepsilon_{1}$ on $C_{1}$. This clearly implies that the function $g: U \rightarrow \mathbb{R}$ defined by

$$
g(x)=\lim _{n \rightarrow \infty} g_{n}(x)
$$

is $C^{\infty}$, strongly convex, and satisfies $|g(x)-f(x)| \leq \varepsilon(x)$ for all $x \in U$. Finally, in order to obtain a real analytic function $g$ with the same properties, one can apply Whitney's theorem on $C^{2}$-fine approximation of $C^{2}$ functions by real analytic functions, as in the last step of the proof of Theorem 1 . 


\section{7. $C^{1}$-Fine APPROXIMATION OF PROPERLY CONVEX FUNCTIONS}

In order to prove Theorem 6 we will have to modify the proof of Theorem 5 by carrying estimates on the derivatives, and take into account the following observation.

Lemma 4. Let $M_{\varepsilon}$ the smooth maximum of Lemma 1, and let $V \subseteq \mathbb{R}^{d}$ be an open set. If $\varphi, \psi \in C^{1}(V)$, then

$$
\left\|D M_{\varepsilon}(\varphi, \psi)-\frac{D \varphi+D \psi}{2}\right\| \leq \frac{1}{2}\|D \varphi-D \psi\| .
$$

Proof. Consider first the one-dimensional case when $\varphi, \psi: V \subseteq \mathbb{R} \rightarrow \mathbb{R}$. We have

$$
\frac{d}{d t} M_{\varepsilon}(\varphi(t), \psi(t))=\frac{\varphi^{\prime}(t)+\psi^{\prime}(t)}{2}+\frac{1}{2} \theta_{\varepsilon}^{\prime}(\varphi(t)-\psi(t))\left(\varphi^{\prime}(t)-\psi^{\prime}(t)\right),
$$

and $\left|\theta_{\varepsilon}^{\prime}(s)\right| \leq 1$ for all $s$ because $\theta_{\varepsilon}$ is 1-Lipschitz. Therefore

$$
\left|\frac{d}{d t} M_{\varepsilon}(\varphi(t), \psi(t))-\frac{\varphi^{\prime}(t)+\psi^{\prime}(t)}{2}\right| \leq \frac{1}{2}\left|\varphi^{\prime}(t)-\psi^{\prime}(t)\right| .
$$

The general case follows at once by considering, for every $x \in V, v \in \mathbb{R}^{d}$ with $\|v\|=1$, the functions $t \mapsto \varphi(x+t v)$ and $t \mapsto \psi(x+t v)$.

Let us now explain the changes one has to make in the proof of Theorem 5 in order to obtain Theorem 6. In this case we do not need to redefine the function $f$ outside $B_{n+2}$ (because we are not going to rely on the proof of Theorem 11), so we simply put $f_{n}=f$ and $\widetilde{f}_{n}=\left(1-r_{n}\right)\left(f_{n}-\beta_{n}\right)+\beta_{n}$. Notice that now we have $f_{n}, \widetilde{f}_{n} \in C^{1}(U)$ for every $n \in \mathbb{N}$.

We use the same preliminaries and Claims 4-6 (with obvious changes) as in the proof of Theorem 5, but in Claim 5 we add

$$
\left\|D \widetilde{f}_{n}-D f_{n}\right\|=\left\|D \widetilde{f}_{n}-D f\right\| \leq \varepsilon_{n} \text { on } B_{n+2},
$$

which clearly holds provided $r_{n}>0$ is small enough. Now we proceed with the inductive construction. Consider the function $\widetilde{f}_{1}$. Notice that $\widetilde{f}_{1}$ is $C^{1}$ on $B_{3} \supset \overline{B_{2}}$. By using the convolutions $\left(f_{1}-\varepsilon_{1}^{\prime} / 2\right) * \delta_{t}$, where $\delta_{t}=t^{-d} \delta(x / t)$, $\delta \geq 0$ being a $C^{\infty}$ function with bounded support and $\int_{\mathbb{R}^{d}} \delta=1$, and taking $t>0$ sufficiently small, we can find a convex function $\varphi_{1} \in C^{\infty}(U)$ of the form $\varphi_{1}=\left(\widetilde{f}_{1}-\varepsilon_{1}^{\prime} / 2\right) * \delta_{t}$ such that $\widetilde{f}_{1}-\varepsilon_{1}^{\prime} \leq \varphi_{1} \leq \widetilde{f}_{1}$ and $\left\|D \varphi_{1}-D \widetilde{f}_{1}\right\| \leq \varepsilon_{1}^{\prime}$ on $\overline{B_{2}}$, where $\varepsilon_{1}^{\prime}:=\frac{1}{2} \min \left\{\varepsilon_{1}, s_{1}\right\}$. Set $g_{1}=\varphi_{1}$.

Claim 9. We have $\left|g_{1}-f\right| \leq 2 \varepsilon_{1}$ and $\left\|D g_{1}-D f\right\| \leq 2 \varepsilon_{1}$ on $C_{1}$.

Proof. We only have to check the second inequality. On $C_{1} \subset B_{2}$ we have

$$
\left\|D g_{1}-D f\right\| \leq\left\|D \varphi_{1}-D \widetilde{f}_{1}\right\|+\left\|D \widetilde{f}_{1}-D f\right\| \leq \varepsilon_{1}^{\prime}+\varepsilon_{1} \leq 2 \varepsilon_{1} .
$$


Now consider $\widetilde{f}_{2}$, and set

$$
\delta_{2}:=\frac{s_{1}}{2}, \text { and } \varepsilon_{2}^{\prime}:=\frac{1}{2} \min \left\{\varepsilon_{2}, s_{2}, \varepsilon_{1}^{\prime}\right\} .
$$

As before, we can find a convex function $\varphi_{2} \in C^{\infty}(U)$ such that $\widetilde{f}_{2}-\varepsilon_{2}^{\prime} \leq$ $\varphi_{2} \leq \widetilde{f}_{2}$ and $\left\|D \varphi_{2}-D \widetilde{f}_{2}\right\| \leq \varepsilon_{2}^{\prime}$ on $\overline{B_{3}}$. Define $g_{2}: U \rightarrow \mathbb{R}$ by

$$
g_{2}(x)= \begin{cases}g_{1}(x), & \text { if } x \in A_{1} \\ M_{\delta_{2}}\left(g_{1}(x), \varphi_{2}(x)\right), & \text { if } x \in U \backslash A_{1} .\end{cases}
$$

Claim 10. The function $g_{2}$ is well defined, convex, $C^{\infty}$, and satisfies

$$
\begin{gathered}
g_{2}=g_{1} \text { on } A_{1}, \\
\left|g_{2}-f\right| \leq 3 \varepsilon_{1} \text { and }\left\|D g_{2}-D f\right\| \leq 5 \varepsilon_{1} \text { on } C_{1}, \\
g_{2}=\varphi_{2} \text { on } U \backslash C_{1}, \text { and } \\
\left|g_{2}-f\right| \leq 2 \varepsilon_{2} \text { and }\left\|D g_{2}-D f\right\| \leq 2 \varepsilon_{2} \text { on } C_{2} \backslash C_{1} .
\end{gathered}
$$

Proof. This time we only have to check the inequalities involving the derivatives. On $A_{1}$ we have $D g_{2}=D g_{1}$, so we have what we need by the preceding claim. On $C_{1} \backslash A_{1}$ we have

$$
\begin{aligned}
& \left\|D g_{1}-D \varphi_{2}\right\| \leq\left\|D g_{1}-D \tilde{f}_{1}\right\|+\left\|D \widetilde{f}_{1}-D f\right\|+\left\|D f-D \widetilde{f}_{2}\right\|+\left\|D \widetilde{f}_{2}-D \varphi_{2}\right\| \leq \\
& \varepsilon_{1}^{\prime}+\varepsilon_{1}+\varepsilon_{2}+\varepsilon_{2}^{\prime} \leq 3 \varepsilon_{1},
\end{aligned}
$$

and therefore, using the preceding lemma,

$$
\begin{aligned}
& \left\|D g_{2}-D f\right\|=\left\|D M_{\delta_{2}}\left(g_{1}, \varphi_{2}\right)-D f\right\| \leq \frac{1}{2}\left\|D g_{1}-D \varphi_{2}\right\|+\frac{1}{2}\left\|D g_{1}+D \varphi_{2}-2 D f\right\| \leq \\
& \frac{3}{2} \varepsilon_{1}+\frac{1}{2}\left\|D g_{1}-D f\right\|+\frac{1}{2}\left\|D \varphi_{2}-D f\right\| \leq \\
& 3 \varepsilon_{1}+\frac{1}{2}\left(\left\|D g_{1}-D \widetilde{f}_{1}\right\|+\left\|D \widetilde{f}_{1}-D f\right\|+\left\|D \varphi_{2}-D \widetilde{f}_{2}\right\|+\left\|D \widetilde{f}_{2}-D f\right\|\right) \leq \\
& 3 \varepsilon_{1}+\frac{1}{2}\left(\varepsilon_{1}^{\prime}+\varepsilon_{1}+\varepsilon_{2}^{\prime}+\varepsilon_{2}\right) \leq 5 \varepsilon_{1} .
\end{aligned}
$$

Finally, on $C_{2} \backslash C_{1}$ we have $g_{2}=\varphi_{2}$, hence

$$
\left\|D g_{2}-D f\right\| \leq\left\|D \varphi_{2}-D \widetilde{f}_{2}\right\|+\left\|D \tilde{f}_{2}-D f\right\| \leq \varepsilon_{2}^{\prime}+\varepsilon_{2} \leq 2 \varepsilon_{2} .
$$

Now consider $\widetilde{f}_{3}$ and put

$$
\delta_{3}:=\frac{s_{2}}{2}, \text { and } \varepsilon_{3}^{\prime}:=\frac{1}{2} \min \left\{\varepsilon_{3}, s_{3}, \varepsilon_{2}^{\prime}\right\},
$$

find a convex function $\varphi_{3} \in C^{\infty}(U)$ such that $\widetilde{f}_{3}-\varepsilon_{3}^{\prime} \leq \varphi_{3} \leq \widetilde{f}_{3}$ and $\left\|D \varphi_{3}-D \widetilde{f}_{3}\right\| \leq \varepsilon_{3}^{\prime}$ on $\overline{B_{4}}$, and define

$$
g_{3}(x)= \begin{cases}g_{2}(x), & \text { if } x \in A_{2} \\ M_{\delta_{3}}\left(g_{2}(x), \varphi_{3}(x)\right), & \text { if } x \in U \backslash A_{2} .\end{cases}
$$


Again, it is not difficult to check that $g_{3}$ is well defined, convex, $C^{\infty}$, and satisfies

$$
\begin{gathered}
g_{3}=g_{2} \text { on } A_{2}, \\
\left|g_{3}-f\right| \leq 3 \varepsilon_{2} \text { and }\left\|D g_{3}-D f\right\| \leq 5 \varepsilon_{2} \text { on } C_{2} \backslash C_{1}, \\
g_{3}=\varphi_{3} \text { on } U \backslash C_{2} \text {, and } \\
\left|g_{3}-f\right| \leq 2 \varepsilon_{3} \text { and }\left\|D g_{3}-D f\right\| \leq 2 \varepsilon_{3} \text { on } C_{3} \backslash C_{2} \text {. }
\end{gathered}
$$

By continuing the inductive process in this manner one can construct a sequence of convex functions $g_{n} \in C^{\infty}(U)$ such that

$$
\begin{gathered}
g_{n+1}=g_{n} \text { on } A_{n}, \\
\left|g_{n+1}-f\right| \leq 3 \varepsilon_{n} \text { and }\left\|D g_{n+1}-D f\right\| \leq 5 \varepsilon_{n} \text { on } C_{n} \backslash C_{n-1}, \\
\left|g_{n+1}-f\right| \leq 2 \varepsilon_{n+1} \text { and }\left\|D g_{n+1}-D f\right\| \leq 2 \varepsilon_{n+1} \text { on } C_{n+1} \backslash C_{n},
\end{gathered}
$$

and with $\left|g_{1}-f\right| \leq 2 \varepsilon_{1} \geq\left\|D g_{1}-D f\right\|$ on $C_{1}$. This clearly implies that the function $g: U \rightarrow \mathbb{R}$ defined by

$$
g(x)=\lim _{n \rightarrow \infty} g_{n}(x)
$$

is $C^{\infty}$, convex, and satisfies $\max \{|g(x)-f(x)|,\|D g(x)-D f(x)\|\} \leq \varepsilon(x)$ for all $x \in U$.

Remark 2. The above proofs more generally show the following: if one has the ability to approximate $C^{1}$ properly convex functions by $C^{\infty}$ strongly convex functions, uniformly on compact sets, and in such a way that the derivatives of the approximations also approximate the derivatives of the given functions, uniformly on compact sets, then one can approximate $C^{1}$ properly convex functions by real analytic strongly convex functions, in the $C^{1}$ fine topology. We will investigate the general problem of uniformly approximating (not properly) convex functions and their derivatives in another paper. These proofs can also be easily adapted to get the following: let $M$ be a (noncompact) Riemannian manifold, and let $\mathcal{P}(M)$ be the class of convex functions $f: M \rightarrow \mathbb{R}$ such that $f(M)$ is an interval of the form $[a, b)$, with $-\infty<a<b \leq \infty$, and for every $\beta \in[a, b)$ the set $f^{-1}[a, \beta]$ is compact. If on $M$ one has the ability to approximate every function of $\mathcal{P}(M)$ by $C^{p}$ convex functions, uniformly on compact sets of $M$, then every function of $\mathcal{P}(M)$ can be approximated by $C^{p}$ convex functions in the $C^{0}$-fine topology. A similar statement holds for $C^{1}$-fine approximation. By combining this observation with [2, Corollary 4.4] we also deduce the following: if $M$ is a complete finite-dimensional Riemannian manifold with sectional curvature $K \leq 0$, then every function in $\mathcal{P}(M)$ can be approximated by $C^{1}$ convex functions in the $C^{0}$-fine topology. The condition that $f$ belong to $\mathcal{P}(M)$ cannot be removed in general, as we already know by considering the case when $M=\mathbb{R}^{n}$, or when $M$ is one of the manifolds constructed in [23]. 


\section{Proofs of Theorems 3 and 4, ANd of Corollaries 4 and 5}

\section{Proof of Theorem 3 .}

Let us first assume that $U=(a, b)$ with $-\infty<a<b<\infty$. If $f\left(a^{+}\right):=$ $\lim _{t \rightarrow a^{+}} f(t)=\infty=\lim _{t \rightarrow b^{-}} f(t):=f\left(b^{-}\right)$then $f$ is proper, so by Theorem 5 we have what we need. If these limits are both finite then we can write $f=c+\ell$, where $\ell(x)=\frac{f\left(b^{-}\right)-f\left(a^{+}\right)}{b-a} x$ is linear, and $c\left(a^{+}\right)=c\left(b^{-}\right)$, so either $c$ is constant, in which case we are done, or else $c$ is proper, and again we conclude by a direct application of Theorem 5.

Thus the only interesting case is when one of these limits is finite and the other one is infinite. Let us assume, for instance, that $\lim _{t \rightarrow a^{+}} f(t)<\infty=$ $\lim _{t \rightarrow b^{-}} f(t)$. There exist $c, d \in(a, b)$ with $c<d$ and $f^{\prime}(d)>f^{\prime}(c)$. Define functions $f_{1}:(a, b) \rightarrow \mathbb{R}$ by

$$
f_{1}(x)= \begin{cases}f(x), & \text { if } a<x \leq d \\ f(d)+f^{\prime}(d)(x-d), & \text { if } d \leq x<b,\end{cases}
$$

and $f_{2}:(-\infty, b) \rightarrow \mathbb{R}$

$$
f_{2}(x)= \begin{cases}f(d)+f^{\prime}(d)(x-d), & \text { if } x \leq d \\ f(x), & \text { if } d \leq x<b .\end{cases}
$$

Notice that $f=\max \left\{f_{1}, f_{2}\right\}$ on $(a, b)$, and that $f_{1}$ and $f_{2}$ are properly convex on $(a, b)$ and $(-\infty, b)$, respectively. Moreover, there exist $\delta>0$ and $x_{1}, x_{2} \in(a, b)$ such that $x_{1}<x_{2}, f_{1}(x) \geq f_{2}(x)+\delta$ for all $x \in\left(a, x_{1}\right]$, and $f_{2}(x)>f_{1}(x)+\delta$ for all $x \in\left[x_{2}, b\right)$. Let $\varepsilon:(a, b) \rightarrow(0, \infty)$ be a continuous function. Put

$$
\varepsilon^{\prime}=\frac{1}{2} \min \left\{\delta, \min _{x \in\left[x_{1}, x_{2}\right]} \varepsilon(x)\right\}
$$

and

$$
\varepsilon_{1}(x)=\frac{1}{2} \min \left\{\varepsilon^{\prime}, \varepsilon(x)\right\}, \quad \varepsilon_{2}(x)= \begin{cases}\varepsilon^{\prime} / 2, & \text { if } x \in\left(-\infty, x_{1}\right] \\ \frac{1}{2} \min \left\{\varepsilon^{\prime}, \varepsilon(x)\right\}, & \text { if } x \in\left[x_{1}, b\right) .\end{cases}
$$

According to the proof of Theorem 5 , we can find strongly convex functions $g_{1}, g_{2} \in C^{\infty}(a, b)$ such that $\left|f_{1}(x)-g_{1}(x)\right| \leq \varepsilon_{1}(x)$ for all $x \in(a, b)$, and $\left|f_{2}(x)-g_{2}(x)\right| \leq \varepsilon_{2}(x)$ for all $x \in(-\infty, b)$. On $(a, b)$ define $g=M_{\varepsilon^{\prime}}\left(g_{1}, g_{2}\right)$, which is a strongly convex $C^{\infty}$ function. We have $g=g_{1}$ on $\left(a, x_{1}\right], g=g_{2}$ on $\left[x_{2}, b\right)$, and $|g(x)-f(x)| \leq \varepsilon(x)$ for every $x \in(a, b)$, as is easily checked. We can now conclude as in the last step of the proof of Theorem 1. The cases when $a=-\infty$ and (or) $b=+\infty$ can be treated in a similar manner.

\section{Proof of Theorem 4.}

It is easy to see that $(i) \Longleftrightarrow($ ii $) \Longrightarrow($ iii $)$. We also have $(i) \Longrightarrow$ $(i v)$ by Theorem 5, and $(i v) \Longrightarrow(v)$ is trivial, so we only have to show $(v) \Longrightarrow(i i i) \Longrightarrow(i i)$. To see $(v) \Longrightarrow(i i i)$, suppose that $f=c \circ P+\ell$ and that $f$ can be $C^{0}$-finely approximated by $C^{p+1}$ convex functions. Let $\varepsilon: \mathbb{R}^{d} \rightarrow(0, \infty)$ be a continuous function such that $\lim _{|x| \rightarrow \infty} \varepsilon(x)=0$. Find 
a convex function $g \in C^{p+1}\left(\mathbb{R}^{d}\right)$ such that $|f-g| \leq \varepsilon$. Then we will see that $f=g$, which contradicts the assumption that $f \notin C^{p+1}\left(\mathbb{R}^{d}\right)$.

Suppose first that there exists $x \in \mathbb{R}^{d}$ such that $g(x)>f(x)$, and take $v \in \operatorname{Ker} P, v \neq 0$. Consider the convex function $h(t)=g(x+t v)-t \ell(v)-$ $f(x)=g(x+t v)-f(x+t v)$, which is defined on $(-\infty, \infty)$. We have $\lim _{|t| \rightarrow \infty}|f(x+t v)-g(x+t v)|=0$, hence also $\lim _{|t| \rightarrow \infty} h(t)=0$. But $h(0)=g(x)-f(x)>0$, and this contradicts the fact that $h$ is convex.

Therefore we must have $f-\varepsilon \leq g \leq f$ on $\mathbb{R}^{d}$. Now assume that there exists $x \in \mathbb{R}^{d}$ such that $g(x)<f(x)$. For the same function $h$ we now have $h(0)=g(x)-f(x)<0=\lim _{|t| \rightarrow \infty} h(t)=0$. By the mean value theorem there exists $t_{0}>0$ such that $h^{\prime}\left(t_{0}\right)>0$, and by convexity $h(t) \geq h(0)+h^{\prime}\left(t_{0}\right) t$ for all $t>0$, which implies $\lim _{t \rightarrow \infty} h(t)=\infty$, a contradiction. Therefore $f=g$ on $\mathbb{R}^{d}$.

Finally, let us check $(i i i) \Longrightarrow($ ii $)$. By Lemma 3 there exists a $(d+1)$ dimensional corner function $C$ which supports $f$ at 0 . And (for every $(d+1)$ dimensional corner function $C$ on $\mathbb{R}^{d}$ ) it is easy to see that there exists a linear functional $\ell: \mathbb{R}^{d} \rightarrow \mathbb{R}$ such that $C(x)-\ell(x)$ tends to $\infty$ as $|x| \rightarrow \infty$. If we set $c=f-\ell$, we have $f(x)=c(x)+\ell(x)$, with $c(x)=f(x)-\ell(x) \geq$ $C(x)-\ell(x) \rightarrow \infty$ as $|x| \rightarrow \infty$.

\section{Proof of Corollary 4}

We may assume $\ell=0$. Denote $V=\operatorname{int}(\mathrm{K})$. Take a $C^{1}$ function $\eta: \mathbb{R}^{n} \rightarrow$ $[0, \infty)$ such that $\eta^{-1}(0)=\mathbb{R}^{n} \backslash V$ and $\eta \leq \varepsilon$ on $V$, use Theorem 5 to find a real analytic strongly convex function $g: V \rightarrow \mathbb{R}$ such that $|f-g| \leq \eta$ on $V$, and define $F: U \rightarrow \mathbb{R}$ by $F=f$ on $U \backslash V$ and $F=g$ on $V$. Let us show that $F$ is convex near $\partial V$. Take $x \in \partial V$ and $v \in \mathbb{R}^{n}$. We have to see that $t \mapsto F(x+t v)$ is convex when $|t|$ is small. If $v$ is tangent to $\partial V$, since $V$ is convex and $F=f$ on $U \backslash V$, we have $F(x+t v)=f(x+t v)$, so this is obvious. If $v$ is transversal to $\partial V$ at $x$, we can assume for instance that there exists $\delta>0$ so that $x+t v \in V$ and $x-t v \in U \backslash V$ for $t \in(0, \delta)$. Define $\varphi_{1}(t)=f(x+t v)$ for $t \in(-\delta, \delta), \varphi_{2}(t)=g(x+t v)$ for $t \in[0, \delta)$, and $\varphi:(-\delta, \delta) \rightarrow \mathbb{R}$ by $\varphi(t)=\varphi_{1}(t)$ if $t<0$ and $\varphi(t)=\varphi_{2}(t)$ if $t>0$. We have to see that $\varphi$ is convex, which amounts to checking that $\varphi_{1}^{\prime}\left(0^{-}\right) \leq \varphi_{2}^{\prime}\left(0^{+}\right)$. And indeed, recalling that $\eta=0$ on $U \backslash V$ and $\frac{d}{d t} \eta(x+t v)_{\mid t=0}=0$, and using convexity of $\varphi_{1}$ on $(-\delta, \delta)$, we have

$$
\begin{aligned}
& \lim _{t \rightarrow 0^{-}} \frac{\varphi_{1}(t)-\varphi_{1}(0)}{t} \leq \lim _{t \rightarrow 0^{+}} \frac{\varphi_{1}(t)-\varphi_{1}(0)}{t} \\
& \leq \lim _{t \rightarrow 0^{+}} \frac{\varphi_{2}(t)-\varphi_{2}(0)+\eta(x+t v)}{t}=\lim _{t \rightarrow 0^{+}} \frac{\varphi_{2}(t)-\varphi_{2}(0)}{t} .
\end{aligned}
$$

To see that $\partial f(x)=\partial F(x)$, take $\zeta \in \partial f(x)$ and assume that $\zeta \notin \partial F(x)$, then there is $v \neq 0$ such that the line $t \mapsto F(x)+t \zeta(v)$ does not support $F(x+t v)$ at $t=0$. As before we may assume that $v$ is transversal to $\partial V$ at $x$ and also, up to replacing $v$ with $-v$, that $x+t v \in V$ and $x-t v \in U \backslash V$ 
for $t \in(0, \delta)$. Let $\varphi_{1}, \varphi_{2}$ be defined as above. We have, for small $s_{1}, s_{2}>0$,

$\frac{F\left(x-s_{1} v\right)-F(x)}{-s_{1}} \leq \zeta(v) \leq \lim _{t \rightarrow 0^{-}} \frac{\varphi_{1}(t)-\varphi_{1}(0)}{t} \leq \lim _{t \rightarrow 0^{+}} \frac{\varphi_{2}(t)-\varphi_{2}(0)}{t} \leq \frac{F\left(x+s_{2} v\right)-F(x)}{s_{2}}$,

which contradicts the assumption that the line $t \mapsto F(x)+t \zeta(v)$ does not support $F(x+t v)$ at $t=0$. Similarly one sees that $\partial F(x) \subseteq \partial f(x)$. Finally, in the case when $f \in C^{1}(U), \partial f(x)$ is a singleton for every $x \in \partial V$, hence so is $\partial F(x)$, and therefore $F$ is differentiable at every point of $\partial V$. Since a differentiable convex function always has a continuous derivative, it follows that $F \in C^{1}(U)$.

The proof of Corollary 5 is easier, and we leave it to the reader's care.

\section{Appendix: Convex functions vs Convex bodies}

In this appendix we recall a (somewhat unbalanced) basic relationship between convex functions and convex bodies, regarding approximation. Given a convex function $f: \mathbb{R}^{d} \rightarrow \mathbb{R}$, if we consider the epigraph $C$ of $f$, which is an unbounded convex body in $\mathbb{R}^{d+1}$, we can approximate $C$ by smooth convex bodies $D_{k}$ such that $\lim _{k \rightarrow \infty} D_{k}=C$ in the Hausdorff distance. Then it is easy to see (via the implicit function theorem) that the boundaries $\partial D_{k}$ are graphs of smooth convex functions $g_{k}: \mathbb{R}^{d} \rightarrow \mathbb{R}$ such that $\lim _{k \rightarrow \infty} g_{k}=f$ uniformly on compact subsets of $\mathbb{R}^{d}$. But when $f$ is not Lipschitz this convergence is not uniform on $\mathbb{R}^{d}$, as the following example shows.

Example 4. Consider the function $f: \mathbb{R} \rightarrow \mathbb{R}, f(x)=x^{2}$. The epigraph $C:=\left\{(x, y): y \geq x^{2}\right\}$ is an unbounded convex body, and the set $D:=$ $\{(x, y): \operatorname{dist}((x, y), C) \leq \varepsilon / 2\}$ is a $C^{1}$ convex body such that $C \subset D \subset$ $C+\varepsilon B$, where $B$ is the unit ball of $\mathbb{R}^{2}$. Hence $D$ approximates $C$ in the Hausdorff distance, and the boundary $\partial D$ is indeed the graph of a $C^{1}$ convex function $g: \mathbb{R} \rightarrow \mathbb{R}$. But the function $g$ does not approximate $f$ on $\mathbb{R}$, because $\lim _{|x| \rightarrow \infty}|f(x)-g(x)|=\infty$.

Therefore one cannot employ results on approximation of (unbounded) convex bodies to deduce results on global approximation of convex functions. By contrast, one can use the well known results on global approximation of Lipschitz convex functions by real analytic convex functions to deduce the following result (first proved by Minkowski in the case when $C$ is bounded):

Theorem 7. Let $C \subset \mathbb{R}^{d}$ be a (not necessarily bounded) convex body. For every $\varepsilon>0$ there exists a real analytic convex body $D$ such that

$$
C \subset D \subset C+\varepsilon B,
$$

where $B$ is the unit ball of $\mathbb{R}^{d}$.

Proof. Consider the 1-Lipschitz, convex function $f: \mathbb{R}^{n} \rightarrow[0, \infty)$ defined by $f(x)=\operatorname{dist}(x, C)$. Using integral convolution with the heat kernel one can produce a real analytic convex (and 1-Lipschitz) function $g: \mathbb{R}^{n} \rightarrow \mathbb{R}$ such that $f-2 \varepsilon / 3 \leq g \leq f-\varepsilon / 3$ on $\mathbb{R}^{n}$. Define $D=g^{-1}(-\infty, 0]$. Since $g$ is 
convex and does not have any minimum on $\partial D=g^{-1}(0)$, we have $\nabla g(x) \neq 0$ for all $x \in \partial D$, hence $\partial D$ is a 1-codimensional real analytic submanifold of $\mathbb{R}^{n}$. Because $f \geq g$, we have $C \subset D$. And if $x \notin C+\varepsilon B$ then $f(x) \geq \varepsilon$, hence $g(x)-\varepsilon / 3 \geq f(x)-\varepsilon \geq 0$, which implies $g(x)>0$, that is $x \notin D$.

\section{REFERENCES}

[1] A.D. Alexandroff, Almost everywhere existence of the second differential of a convex function and some properties of convex surfaces connected with it, Leningrad State Univ. Annals (Uchenye Zapiski) Math. Ser. 6, (1939). 3-35.

[2] D. Azagra, and J. Ferrera, Inf-convolution and regularization of convex functions on Riemannian manifolds of nonpositive curvature, Rev. Mat. Complut. 19 (2006), no. $2,323-345$.

[3] D. Azagra, J. Ferrera, F. López-Mesas, Nonsmooth analysis and Hamilton-Jacobi equations on Riemannian manifolds, J. Funct. Anal. 220 (2005), no. 2, 304-361.

[4] V. Bangert, Analytische Eigenschaften konvexer Funktionen auf Riemannschen Mannigfaltigkeiten, J. Reine Angew. Math. 307/308 (1979), 309-324.

[5] V. Bangert, Über die Approximation von lokal konvexen Mengen, Manuscripta Math. 25 (1978), no. 4, 397-420.

[6] F. H. Clarke, Yu. S. Ledyaev, R. J. Stern, P. R. Wolenski, Nonsmooth analysis and control theory, Graduate Texts in Mathematics, 178. Springer-Verlag, New York, 1998.

[7] J. Cheeger, and D. Gromoll, On the structure of complete manifolds of nonnegative curvature, Ann. of Math. 96 (1972), 413-443.

[8] R. Deville, V. Fonf, P. Hájek, Analytic and $C^{k}$ approximations of norms in separable Banach spaces, Studia Math. 120 (1996), no. 1, 61-74.

[9] R. Deville, V. Fonf, P. Hájek, Analytic and polyhedral approximation of convex bodies in separable polyhedral Banach spaces, Israel J. Math. 105 (1998), 139-154.

[10] M. Ghomi, The problem of optimal smoothing for convex functions. Proc. Amer. Math. Soc. 130 (2002), no. 8, 2255-2259.

[11] M. Ghomi, Optimal smoothing for convex polytopes. Bull. London Math. Soc. 36 (2004), no. 4, 483-492

[12] R. E. Greene, and K. Shiohama, Convex functions on complete noncompact manifolds: topological structure, Invent. Math. 63 (1981), no. 1, 129-157.

[13] R. E. Greene, and K. Shiohama, Convex functions on complete noncompact manifolds: differentiable structure, Ann. Sci. École Norm. Sup. (4) 14 (1981), no. 4, 357-367 (1982).

[14] R. E. Greene, and H. Wu, On the subharmonicity and plurisubharmonicity of geodesically convex functions, Indiana Univ. Math. J. 22 (1972/73), 641-653.

[15] R. E. Greene, and $\mathrm{H}$. Wu, $C^{\infty}$ convex functions and manifolds of positive curvature, Acta Math. 137 (1976), no. 3-4, 209-245.

[16] R. E. Greene, and H. Wu, $C^{\infty}$ approximations of convex, subharmonic, and plurisubharmonic functions, Ann. Sci. École Norm. Sup. (4) 12 (1979), no. 1, 47-84.

[17] D. Gromoll, and W. Meyer, On complete open manifolds of positive curvature, Ann. of Math. 90 (1969) 75-90.

[18] P. Gruber, Aspects of approximation of convex bodies. Handbook of convex geometry, Vol. A, B, 319-345, North-Holland, Amsterdam, 1993.

[19] H. Rademacher Über partielle und totale Differenzierbarkeit I., Math. Ann. 89 (1919), 340-359.

[20] R.T. Rockafellar, Convex analysis. Princeton Mathematical Series, No. 28. Princeton University Press, Princeton, N.J., 1970 
[21] T. Sakai, Riemannian Geometry, Translations of Mathematical Monographs, 149. American Mathematical Society, Providence, RI, 1996.

[22] R. Schneider, Convex bodies: the Brunn-Minkowski theory. Encyclopedia of Mathematics and its Applications, 44. Cambridge University Press, Cambridge, 1993.

[23] P. A. N. Smith, Counterexamples to smoothing convex functions, Canad. Math. Bull. 29 (1986), no. 3, 308-313.

[24] T. Strömberg, The operation of infimal convolution, Dissertationes Math. (Rozprawy Mat.) 352 (1996)

[25] H. Whitney, Analytic extensions of differential functions in closed sets, Trans. Amer. Math. Soc. 36 (1934), 63-89.

iCMAT (CSIC-UAM-UC3-UCM), Departamento de AnÁlisis Matemático, FaCultad Ciencias Matemáticas, Universidad Complutense, 28040, Madrid, Spain

E-mail address: daniel_azagra@mat.ucm.es 